\title{
The Malliavin calculus for processes with conditionally independent increments
}

\author{
Aleh L. Yablonski \\ Department of Functional Analysis, Belarusian State University, F.Skaryna av.,4, \\ 220050, Minsk, Belarus, yablonski@bsu.by. Supported by INTAS grant 03-55-1861
}

Summary. The purpose of this paper is to construct the analog of Malliavin derivative $D$ and Skorohod integral $\delta$ for some class of processes which include, in particular, processes with conditionally independent increments. We introduce the family of orthogonal polynomials. By using these polynomials it is proved the chaos decomposition theorem of $L^{2}(\Omega)$. The definition of Malliavin derivative and Skorohod integral for a certain class of stochastic processes is given and it is shown that they are equal respectively to the annihilation and the creation operators on the Fock space representation of $L^{2}(\Omega)$. The analogue of Clark-Haussmann-Ocone formula for processes with conditionally independent increments is also established.

Key words and phrases: processes with conditionally independent increments, Malliavin calculus, Skorohod integral, multiple integral, orthogonal polynomials, chaos expansion, Clark-Haussmann-Ocone formula.

\section{Introduction}

It was shown by Karatzas and Ocone [14] how the stochastic calculus of variations developed by Malliavin [20] can be used in mathematical finance. This discovery led to an increase in the interest in the Malliavin calculus.

In the Brownian setup the calculus of variations has a complete form (see the elegant presentation of Nualart [22]). It is based on the operators $D$ and $\delta$ which are called Malliavin derivative and Skorohod integral, respectively. There are two equivalent approaches to definition of the operator $D$ : as a variational derivative and through the chaos decomposition.

For discontinuous processes it is possible to develop the Malliavin-type calculus by using some "generalized" or "weak" derivatives (see, e.g., [1, 4, 15] and references therein). Nevertheless, it was shown in [24] that in the Poisson case small perturbations of the trajectories lead to a certain difference operator. This idea was extended for Lévy processes in [26, 28, 31, 33]. 
Alternatively, the operator $D$ can be defined by its action on the chaos representation of $L^{2}$-functionals. The case of normal martingales with chaotic representation property was considered in [19]. But, in general, a Lévy process has no chaotic representation property in the sense that Brownian motion and Poisson process do. There are two different chaotic expansions introduced in [11] and [23]. By using these expansions two types of Malliavin operators for some classes of Lévy processes have been studied in the papers $[2,7,8,16$, $18,25,31]$. The relationship between them has been shown in $[2,31]$. The connection of such derivative to the difference operator from $[26,28]$ was studied in $[18,31,33]$. For random Lévy measures the Skorohod integral and Malliavin derivative were considered in $[5,6]$.

The purpose of the paper is to construct the Malliavin calculus for some class of processes which includes, in particular, the processes with conditionally independent increments. It is also proved the chaos representation theorem for such type of processes.

In general the processes with conditionally independent increments can be described in terms of their triplets of characteristics $(B, \mu, \nu)$, where $B$ represents the "drift" part, $\mu$ is connected with continuous martingale part (Gaussian part for Lévy processes) and $\nu$ is a compensator of measure associated to the jumps of the original process. Since $B$ is a bounded variation process then for our purposes we can set $B=0$ without loss of generality. Therefore we start with two random measures $\mu$ and $\nu$ on certain measurable spaces which describe, respectively, the continuous and discontinuous parts of the process. In Section 2 we define the appropriate Hilbert space $H$ connected to these measures and stochastic process indexed by elements of $H$. This construction allows us to consider aa rather general class of processes. The system of generalized orthogonal polynomials defined in [33] is used in the proof of the chaos decomposition of $L^{2}$ functionals.

Section 3 deals with multiple integrals with respect to the $L^{2}$-valued measure generated by the considering process. Their connection to generalized orthogonal polynomials and chaos expansion is also proved.

In Sections 4 and 5 we define the operator $D$ and its adjoint operator $\delta$. Then we show that they are generalizations of the Malliavin derivative and Skorohod integral. It is also proved that their action on the Fock space representation of $L^{2}$-functionals coincides with annihilation and creation operators. In the end of the last section we prove the analogue of Clark-HaussmannOcone formula for processes with conditionally independent increments.

\section{The chaos decomposition}

Let $(\Omega, \mathcal{F}, \mathrm{P})$ be a complete probability space. Suppose that $\mu$ and $\nu$ are random measures defined on the measurable spaces $(T, \mathcal{A})$ and $\left(T \times X_{0}, \mathcal{B}\right)$ respectively, such that the following conditions are satisfied:

1. $\mu(A, \cdot)$ and $\nu(B, \cdot)$ are $\mathcal{F}$-measurable for all $A \in \mathcal{A}$ and $B \in \mathcal{B}$, 
2. $\mu(\cdot, \omega)$ and $\nu(\cdot, \omega)$ are $\sigma$-finite measures without atoms for all $\omega \in \Omega$.

Consider $\Delta \notin X_{0}$ and denote $X=X_{0} \cup\{\Delta\}, \mathcal{G}=\sigma(\mathcal{A} \times\{\Delta\}, \mathcal{B})$. Define a new measure $\pi(d t d x)=\mu(d t) \delta_{\Delta}(d x)+\nu\left(d t d x \cap\left(T \times X_{0}\right)\right)$ on the $\sigma$-algebra $\mathcal{G}$. Here $\delta_{\Delta}(d x)$ is the measure which gives mass one to the point $\Delta$. Let $\mathcal{H}$ be a $\sigma$-algebra generated by measure $\pi$ and the collection $\mathcal{N}$ of P-null events of $\mathcal{F}$, i.e.,

$$
\mathcal{H}=\sigma\{\pi(A): A \in \mathcal{G}\} \vee \mathcal{N} .
$$

Define a new measure $M_{\pi}$ on $\sigma$-algebra $\mathcal{G} \otimes \mathcal{F}$ in the following way. For any $A \in \mathcal{G}$ and $B \in \mathcal{F}$ we set $M_{\pi}(A \times B)=\mathrm{E}\left[\mathbf{1}_{B} \pi(A)\right]$. Then extension of it on the $\sigma$-algebra $\mathcal{G} \otimes \mathcal{F}$ can be done as usual (see e.g., [27, Ch. 4]). Suppose that measure $M_{\pi}$ is $\sigma$-finite. Then there exists the sequence of the sets $U_{n} \in \mathcal{G} \otimes \mathcal{F}$ such that $\bigcup_{n=1}^{\infty} U_{n}=T \times X \times \Omega$ and $M_{\pi}\left(U_{n}\right)<\infty$. We can choose $U_{n}=A_{n} \times B_{n}$, where $A_{n} \in \mathcal{G}$ and $B_{n} \in \mathcal{F}$. Indeed for any $\epsilon>0$ and each $U_{n}$ we can find $A_{n}^{k} \in \mathcal{G}$ and $B_{n}^{k} \in \mathcal{F}$ such that $U_{n} \subset \bigcup_{k=1}^{\infty} A_{n}^{k} \times B_{n}^{k}$ and $M_{\pi}\left(U_{n}\right) \leq \sum_{k=1}^{\infty} M_{\pi}\left(A_{n}^{k} \times B_{n}^{k}\right) \leq M_{\pi}\left(U_{n}\right)+\epsilon$. Hence $M_{\pi}\left(A_{n}^{k} \times B_{n}^{k}\right)<\infty$ and $T \times X \times \Omega=\bigcup_{k, n=1}^{\infty} A_{n}^{k} \times B_{n}^{k}$. Renumeration of the sets $A_{n}^{k} \times B_{n}^{k}$ yields the desired result.

If we consider the restriction of the measure $M_{\pi}$ on the $\sigma$-algebra $\mathcal{G} \otimes \mathcal{H}$ then it is possible to show that it will be $\sigma$-finite. Indeed, let $U_{n}=A_{n} \times B_{n}$, $A_{n} \in \mathcal{G}$ and $B_{n} \in \mathcal{F}$ be as above then $\bigcup_{n=1}^{\infty} A_{n}=T \times X$ and $\bigcup_{n=1}^{\infty} B_{n}=\Omega$. Denote by $C_{n}^{m}$ the following sets: $C_{n}^{m}=\left\{\omega \in \Omega: \pi\left(A_{n}\right) \leq m\right\}, m=1,2, \ldots$ Then $C_{n}^{m} \in \mathcal{H}$ and $\bigcup_{m=1}^{\infty} C_{n}^{m}=\left\{\pi\left(A_{n}\right)<\infty\right\} \supset\left(B_{n} \backslash N_{n}\right)$, where $\mathrm{P}\left(N_{n}\right)=0$. Hence $\bigcup_{n, m=1}^{\infty} A_{n} \times\left(C_{n}^{m} \cup N_{n}\right)=T \times X \times \Omega$ and $M_{\pi}\left(A_{n} \times\left(C_{n}^{m} \cup N_{n}\right)\right) \leq m<$ $\infty$. In fact we have a stronger property: $\pi\left(A_{n}, \omega\right)<\infty$ for all $\omega \in \bigcup_{m=1}^{\infty} C_{n}^{m}$. This property implies the sigma-finiteness of the $\pi$ in the sense [27, Ch. 4 , Def. 21].

Consider the Hilbert space $H=L^{2}\left(T \times X \times \Omega, \mathcal{G} \otimes \mathcal{H}, M_{\pi}\right)$ and assume that it is separable. Denote by $\pi(f)$ the integral of $f$ with respect to measure $\pi$ :

$$
\pi(f)=\int_{T \times X} f(t, x) \pi(d t d x) .
$$

It was shown in $[27$, Ch. 4] that if $\mathrm{E}[\pi(|f|)]<\infty$ then $\pi(f)$ is $\mathcal{H}$-measurable or $\mathcal{F}$-measurable whenever $f$ is $\mathcal{G} \otimes \mathcal{H}$-measurable or $\mathcal{G} \otimes \mathcal{F}$-measurable respectively. The scalar product and the norm in $H$ will be denoted by $\langle\cdot ; \cdot\rangle_{H}$ and $\|\cdot\|_{H}$ respectively, i.e. for any $f, g \in H$

$$
\langle f ; g\rangle_{H}=\mathrm{E}(\pi(h g))=\mathrm{E} \int_{T \times X} h(t, x) g(t, x) \pi(d t d x),\|f\|_{H}^{2}=\mathrm{E}\left(\pi\left(h^{2}\right)\right) .
$$

Definition 1. We say that a stochastic process $L=\{L(h), h \in H\}$ is a conditional additive process on $H$ if the following conditions are satisfied.

1. For all $h, g \in H$ and $\alpha, \beta \in L^{\infty}(\Omega, \mathcal{H}, \mathrm{P})$ we have P-a.s.

$$
L(\alpha h+\beta g)=\alpha L(h)+\beta L(g),
$$


2. For all $z \in \mathbb{R}$ and $h \in H$

$$
\begin{aligned}
\mathrm{E}\left[e^{i z L(h)} \mid \mathcal{H}\right]=\exp ( & -\frac{1}{2} z^{2} \int_{T} h^{2}(t, \Delta) \mu(d t) \\
& \left.+\int_{T \times X_{0}}\left(e^{i z h(t, x)}-1-i z h(t, x)\right) \nu(d t d x)\right)
\end{aligned}
$$

Remark 2. 1. In this definition we suppose that the process $L(h)$ can be defined on the original probability space $(\Omega, \mathcal{F}, \mathrm{P})$. If it is not the case then it is possible to define $\mu, \nu$, and $L(h)$ verifying the above conditions on some extension $\left(\Omega^{\prime}, \mathcal{F}^{\prime}, \mathrm{P}^{\prime}\right)$ of the original probability space. So we can always assume that original probability space $(\Omega, \mathcal{F}, \mathrm{P})$ is rich enough for defining all necessary objects.

2 . The definition 1 shows that the random variable $L(h)$ has a conditionally infinitely divisible distribution.

3. If measure $\nu$ is zero and measure $\mu$ is deterministic then $L$ is an isonormal Gaussian process (see, e.g., [22, Def. 1.1.1, p. 4]).

4. If measures $\mu$ and $\nu$ are deterministic then $L$ is an isonormal Lévy process (see, e.g., [33]).

Example 3. Let $L_{t}, t \geq 0$ be a càdlàg real-valued process with $\mathcal{H}$-conditionally independent increments on complete probability space $(\Omega, \mathcal{F}, \mathrm{P})$, where $\mathcal{H} \subset$ $\mathcal{F}$. Suppose that $L_{t}$ is a quasi-left-continuous semimartingale with respect to filtration $\mathcal{F}_{t}, t \geq 0$ generated by the natural filtration and $\sigma$-algebra $\mathcal{H}$, i.e. $\mathcal{F}_{t}=\bigcap_{s>t}\left(\mathcal{F}_{t}^{0} \vee \mathcal{H}\right)$, where $\mathcal{F}_{t}^{0}=\sigma\left\{L_{s}: s \leq t\right\}$. In this case there exists a version of the characteristics $(B, \mu, \nu)$ of $L_{t}$ (see, e.g., $\left.[12,17]\right)$ such that:

1. $B_{t}, t \geq 0$ is a continuous process of locally bounded variation with $B_{0}=0$;

2. $\mu_{t}, t \geq 0$ is a continuous nondecreasing process with $\mu_{0}=0$;

3. $\nu(d t d x, \omega)$ is a predictable random measure defined on the Borel $\sigma$-algebra of $\mathbb{R}_{+} \times \mathbb{R}_{0}$, where $\mathbb{R}_{0}=\mathbb{R} \backslash\{0\}$ such that $\nu\left(\{t\} \times \mathbb{R}_{0}\right)=0, \int_{0}^{t} \int_{\mathbb{R}_{0}}\left(|x|^{2} \wedge\right.$ 1) $\nu(d s d x)<\infty$ for all $t \geq 0 \mathrm{P}$ a.s.

Moreover, $B, \mu$ and $\nu$ are $\mathcal{H}$-measurable and we have for all $z \in \mathbb{R}$ and $s \leq t$

$$
\begin{gathered}
\mathrm{E}\left[\exp \left(i z\left(L_{t}-L_{s}\right)\right) \mid \mathcal{H}\right] \\
=\exp \left[i z\left(B_{t}-B_{s}\right)-\frac{1}{2} z^{2}\left(\mu_{t}-\mu_{s}\right)+\int_{s}^{t} \int_{\mathbb{R}_{0}}\left(e^{i z x}-1-i z x \mathbf{1}_{|x| \leq 1}\right) \nu(d t d x)\right] .
\end{gathered}
$$

Here $\mu_{t}=\left\langle L^{c} ; L^{c}\right\rangle_{t}$ is a quadratic variation of the continuous parts of $L, \nu$ is a compensator of the random measure $N(d t d x)$ associated to the jumps of $L$. Hence the following canonical representation holds:

$$
L_{t}=L_{0}+L_{t}^{c}+\int_{0}^{t} \int_{|x| \leq 1} x(N(d s d x)-\nu(d s d x))+\int_{0}^{t} \int_{|x|>1} x N(d s d x)+B_{t} .
$$


We can define $L^{2}$-valued measure $L(d t d x)$ with conditionally independent values on the disjoint sets by

$$
L(A)=\int_{A(0)} d L_{t}^{c}+\iint_{A \backslash A(0)}(N(d t d x)-\nu(d t d x)),
$$

where $A \in \mathcal{G}, M_{\pi}(A)<\infty, A(0)=A \cap(T \times\{0\})$.

Suppose that $T=\mathbb{R}_{+}, X_{0}=\mathbb{R}_{0}, \Delta=0$. Let $\mathcal{A}$ and $\mathcal{B}$ be the Borel $\sigma$ algebras of $T$ and $T \times X_{0}$ respectively. Let $\mu(d t)$ be the measure on $\mathcal{A}$ generated by process $\mu_{t}$. Suppose that $\sigma$-algebra $\mathcal{H}$ is generated by the measures $\mu$ and $\nu$. Construct measure $\pi$ and Hilbert space $H=L^{2}\left(\mathbb{R}_{+} \times \mathbb{R} \times \Omega, \mathcal{G} \otimes \mathcal{H}, M_{\pi}\right)$ as above. Then it is easy to show that for any $h \in H$ the random variable

$$
\begin{aligned}
L(h)=\int_{0}^{\infty} h(s, 0) d L_{s}^{c}+\int_{0}^{\infty} \int_{\mathbb{R}_{0}} h(s, x)(N( & d s d x)-\nu(d s d x)) \\
& =\iint_{\mathbb{R}_{+} \times \mathbb{R}} h(t, x) L(d t d s)
\end{aligned}
$$

is well defined and $L(h)$ is a conditional additive process on $H$.

On the other hand if we have a conditional additive process $L(h)$ on $H$ then the $L^{2}$-valued measure $L(d t d x)$ which is given by $L(A)=L\left(\mathbf{1}_{A}\right)$ for all $A \in \mathcal{G}$ with $M_{\pi}(A)<\infty$ has conditionally independent values on the disjoint sets. In order to express the process $L_{t}$ in terms of process $L(h)$ we can not write $L_{t}=$ $L\left(h_{t}\right)$, where $h_{t}(s, x)=\mathbf{1}_{[0 ; t]}(s) \mathbf{1}_{\{0\}}(x)+x \mathbf{1}_{[0 ; t]}(s)$ because, in general, $h_{t} \notin H$. Therefore we define for any $n \geq 1$ the random variable $\tau_{n}=\inf \left\{t>0: \mu_{t} \leq\right.$ $n\}$. Obviously $\tau_{n}$ is an increasing sequence and $h_{t \wedge \tau_{n}} \mathbf{1}_{\{x=0\}} \in H$ for all $t \geq 0$ and $n \geq 1$. Moreover, the process $L\left(h_{t \wedge \tau_{n}} \mathbf{1}_{\{x=0\}}\right), t \geq 0$ has a version with continuous sample paths and $L\left(h_{t \wedge \tau_{n}} \mathbf{1}_{\{x=0\}}\right)=L\left(h_{t \wedge \tau_{m}} \mathbf{1}_{\{x=0\}}\right)$ if $t \leq \tau_{n}$ and $n<m$. Therefore $L^{c}(t)=\lim _{n \rightarrow \infty} L\left(h_{t \wedge \tau_{n}} \mathbf{1}_{\{x=0\}}\right)$ well defined continuous process. Furthermore for any set $A \in \mathcal{B}$ such that $M_{\pi}(A)=\mathrm{E}[\nu(A)]<\infty$ the random variable $N(A)=L\left(\mathbf{1}_{A}\right)+\nu(A)$ is an integer valued random measure with compensator measure $\nu$. And we can define

$$
\bar{L}_{t}=L^{c}(t)+\int_{0}^{t} \int_{|x| \leq 1} x(N(d s d x)-\nu(d s d x))+\int_{0}^{t} \int_{|x|>1} x N(d s d x) .
$$

Comparing equalities (2) and (4) we deduce that the only characteristics which cannot be determined from $L(h)$ is the drift process $B$ and initial value $L(0)$.

Denote by $K$ the following subset of $H$ :

$$
K=\left\{h \in H: h \mathbf{1}_{X_{0}} \in L^{\infty}\left(T \times X \times \Omega, \mathcal{G} \otimes \mathcal{H}, M_{\pi}\right), \pi\left(h^{2}\right) \in L^{\infty}(\Omega, \mathcal{H}, \mathrm{P})\right\}
$$

The elements of $K$ satisfy the following properties.

Lemma 4. Suppose that $h \in K$ then 
1. $|h| \in K$ and $z h \in K$ for all $z \in \mathbb{R}$.

2. $\mathrm{E}\left[\exp \left(\pi\left(h^{2}\right) / 2\right)\right]<\infty, \mathrm{E}\left[\pi\left(|h|^{k} \mathbf{1}_{X_{0}}\right)\right]<\infty$ and $\mathrm{E}\left[\pi\left(h^{2}\right)^{k}\right]<\infty$ for all $k \geq 2$.

3. $\mathrm{E}\left[\exp \left(\frac{1}{2} \int_{T} h^{2}(t, \Delta) \mu(d t)+\int_{T \times X_{0}}\left(e^{h(t, x)}-1-h(t, x)\right) \nu(d t d x)\right)\right]<\infty$.

Proof. The first two properties are evident. The proof of the last statement is based on the boundedness of $h \mathbf{1}_{X_{0}}$ and inequality $\left|e^{x}-1-x\right| \leq e^{|x|} x^{2} / 2$, which can be proved by using Taylor's formula. We omit the details.

Lemma 5. The set $K$ is dense in $H$.

Proof. Choose a $h \in H$. Then $\pi\left(h^{2}\right)<\infty$ a.s. Consider two sequences of sets $B_{m}=\left\{\pi\left(h^{2}\right) \leq m\right\} \in \mathcal{H}$ and $C_{k}=\{(t, x, \omega):|h(t, x, \omega)| \leq k\} \in \mathcal{G} \otimes \mathcal{H}$. Denote $h_{k, m}=h \mathbf{1}_{C_{k}} \mathbf{1}_{B_{m}}$ It is evident that $h_{k, m} \in K$ for all integers $k, m \geq 1$. By dominated convergence theorem we have $\left\|h_{k, m}-h \mathbf{1}_{B_{m}}\right\|_{H} \rightarrow 0$ as $k \rightarrow \infty$ and $\left\|h \mathbf{1}_{B_{m}}-h\right\|_{H}=\mathrm{E}\left[\pi\left(h^{2}\right) \mathbf{1}_{\Omega \backslash B_{m}}\right] \rightarrow 0$ as $m \rightarrow \infty$ which completes the proof of the lemma.

The following lemma describes some properties of $L(h)$.

\section{Lemma 6.}

1. If $h \in H$ then $L(h) \in L^{2}(\Omega, \mathcal{F}, \mathrm{P})$. Furthermore $\mathrm{E}[L(h) \mid \mathcal{H}]=0$ and $\mathrm{E}[L(h) L(g) \mid \mathcal{H}]=\pi(h g)$.

2. Let $h_{n} \in H$ be a sequence such that $\left\|h_{n}-h\right\|_{H} \rightarrow 0$ as $n \rightarrow \infty$ for some $h \in H$. Then $\mathrm{E}\left[\left(L\left(h_{n}\right)-L(h)\right)^{2} \mid \mathcal{H}\right] \rightarrow 0$ as $n \rightarrow \infty$ in $L^{1}(\Omega, \mathcal{F}, \mathrm{P})$.

3. If $h \in K$ then $L(h) \in L^{p}(\Omega, \mathcal{F}, \mathrm{P})$ for all $p \geq 1, \mathrm{E}[\exp (c|L(h)|)]<\infty$ for all $c \in \mathbb{R}$, and

$$
\begin{aligned}
& \mathrm{E}[\exp (L(h)) \mid \mathcal{H}]= \\
& \quad \exp \left(\frac{1}{2} \int_{T} h^{2}(t, \Delta) \mu(d t)+\int_{T \times X_{0}}\left(e^{h(t, x)}-1-h(t, x)\right) \nu(d t d x)\right) .
\end{aligned}
$$

4. Let $h_{1}, \ldots h_{n} \in K$ be such that $h_{i} h_{j}=0 M_{\pi}$-a.s. if $i \neq j$. Then for any integers $p_{1}, \ldots, p_{n} \geq 1$ we have

$$
\mathrm{E}\left[L\left(h_{1}\right)^{p_{1}} \cdots L\left(h_{n}\right)^{p_{n}} \mid \mathcal{H}\right]=\mathrm{E}\left[L\left(h_{1}\right)^{p_{1}} \mid \mathcal{H}\right] \cdots \mathrm{E}\left[L\left(h_{n}\right)^{p_{n}} \mid \mathcal{H}\right] .
$$

Proof. 1. Choose a $B \in \mathcal{H}, \mathrm{P}(B)>0$. Denote by $f_{B}(z)$ the characteristic function of the random variable $L(h)$ with respect to restriction of probability on the set $B$, i.e., $f_{B}(z)=\mathrm{E}\left[\mathbf{1}_{B} e^{i z L(h)}\right]$. Equality (1) implies that $f_{B}(z)$ can be written in the following form:

$$
\begin{aligned}
f_{B}(z)=\mathrm{E}\left[\mathbf { 1 } _ { B } \operatorname { e x p } \left(-\frac{z^{2}}{2} \int_{T} h^{2}(t, \Delta) \mu(d t)\right.\right. \\
\left.\left.\quad+\int_{T \times X_{0}}\left(e^{i z h(t, x)}-1-i z h(t, x)\right) \nu(d t d x)\right)\right] .
\end{aligned}
$$


By using this equality it is easy to show that $f_{B}(z)$ two times differentiable function. Hence $L(h) \in L^{2}(\Omega, \mathcal{F}, \mathrm{P})$. Taking the first and second derivatives at $z=0$ in both sides of the equality above yields $\mathrm{E}\left[L(h) \mathbf{1}_{B}\right]=0$ and $\mathrm{E}\left[L(h)^{2} \mathbf{1}_{B}\right]=\mathrm{E}\left[\pi\left(h^{2}\right) \mathbf{1}_{B}\right]$. Then $\mathrm{E}[L(h) \mid \mathcal{H}]=0, \mathrm{E}\left[L(h)^{2} \mid \mathcal{H}\right]=\pi\left(h^{2}\right)$ and $\mathrm{E}[L(h) L(g) \mid \mathcal{H}]=\mathrm{E}\left[L(h+g)^{2}-L(h-g)^{2} \mid \mathcal{H}\right] / 4=\pi(h g)$ which completes the proof of the first statement of the lemma.

2. Suppose that $h_{n} \rightarrow h$ as $n \rightarrow \infty$ in $H$. It means that $\mathrm{E}\left(\pi\left(\left(h_{n}-h\right)^{2}\right)\right) \rightarrow$ 0 as $n \rightarrow \infty$. Then from the first part of the lemma we have $\mathrm{E}\left[\left(L\left(h_{n}\right)-\right.\right.$ $\left.L(h))^{2} \mid \mathcal{H}\right]=\pi\left(\left(h_{n}-h\right)^{2}\right)$ which implies the proof of second statement of the lemma.

3. Denote by $u(z)$ the following expression:

$$
\begin{aligned}
u(z)=\mathrm{E}\left[\operatorname { e x p } \left(\frac{z^{2}}{2} \int_{T} h^{2}(t, \Delta) \mu(d t)\right.\right. & \\
& \left.\left.+\int_{T \times X_{0}}\left(e^{z h(t, x)}-1-z h(t, x)\right) \nu(d t d x)\right)\right] .
\end{aligned}
$$

Since $h \in K$ then Lemma 4 implies that $u(z)$ is finite for all $z \in \mathbb{R}$. The right hand side in the equality above is meaningful even $z$ is complex. Indeed if $z=a+i b$ then $\operatorname{Re}\left(e^{z h}-1-z h\right)=e^{a h} \cos (b h)-1-a h=\left(e^{a h}-1-a h\right)+$ $e^{a h}(\cos (b h)-1) \leq\left(e^{a h}-1-a h\right)$. Hence from Lemma 4 we have

$$
\begin{aligned}
\mid \mathrm{E}\left[\operatorname { e x p } \left(\frac{z^{2}}{2} \int_{T} h^{2}(t,\right.\right. & \left.\left.\Delta) \mu(d t)+\int_{T \times X_{0}}\left(e^{z h(t, x)}-1-z h(t, x)\right) \nu(d t d x)\right)\right] \mid \\
\leq & \mathrm{E}\left[\operatorname { e x p } \left(\frac{a^{2}}{2} \int_{T} h^{2}(t, \Delta) \mu(d t)\right.\right. \\
& \left.\left.+\int_{T \times X_{0}}\left(e^{a h(t, x)}-1-a h(t, x)\right) \nu(d t d x)\right)\right]<\infty .
\end{aligned}
$$

The function $u(z)$ is analytic function for all $z \in \mathbb{C}$. If $z=i t, t \in \mathbb{R}$ then $u(z)=f(t)=\mathrm{E}\left[e^{i t L(h)}\right]$ coincides with characteristic function of $L(h)$. Hence characteristic function $f(t)$ infinitely differentiable for all $t \in \mathbb{R}$ and $L(h)$ has finite moments of all orders, i.e. $L(h) \in L^{p}(\Omega, \mathcal{F}, \mathrm{P})$ for all $p \geq 1$. Moreover

$$
u(z)=\sum_{k=0}^{\infty} \frac{1}{k !} z^{k} \mathrm{E}\left[L(h)^{k}\right],
$$

where the radius of convergence of the series being infinite. It follows that

$$
\mathrm{E}[\exp (c|L(h)|)]=\sum_{k=0}^{\infty} \frac{c^{k}}{k !} \mathrm{E}\left[|L(h)|^{k}\right]<\infty
$$

for all $c \in \mathbb{R}$. Hence $f(z)=\mathrm{E}[\exp (z L(h))]$ is analytic for all complex $z$. The uniqueness theorem yields $u(z)=f(z)$ which implies the third statement of the lemma. 
4. From the previous parts of the lemma we have $L\left(h_{k}\right) \in L^{p}(\Omega, \mathcal{F}, \mathrm{P})$ for all $p \geq 1$ and conditional characteristic function of random variables $L\left(h_{k}\right)$ is infinitely differentiable if $h_{1}, \ldots h_{n} \in K$. Since $h_{i} h_{j}=0 M_{\pi}$-a.s. if $i \neq j$ then we have the following equality:

$$
\begin{gathered}
\mathrm{E}\left[\exp \left(i \sum_{k=1}^{n} z_{k} L\left(h_{k}\right)\right) \mid \mathcal{H}\right]=\exp \left(\frac{1}{2} \sum_{k=1}^{n} z_{k}^{2} \int_{T} h_{k}^{2}(t, \Delta) \mu(d t)\right. \\
\left.+\sum_{k=1}^{n} \int_{T \times X_{0}}\left(e^{i z_{k} h_{k}(t, x)}-1-i z_{k} h_{k}(t, x)\right) \nu(d t d x)\right)= \\
\mathrm{E}\left[\exp \left(i z_{1} L\left(h_{1}\right)\right) \mid \mathcal{H}\right] \cdots \mathrm{E}\left[\exp \left(i z_{n} L\left(h_{n}\right)\right) \mid \mathcal{H}\right]
\end{gathered}
$$

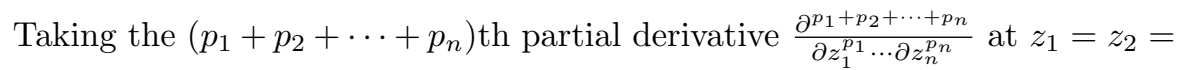
$\cdots=0$ in both sides of the above equality yields

$$
\mathrm{E}\left[L\left(h_{1}\right)^{p_{1}} \cdots L\left(h_{n}\right)^{p_{n}} \mid \mathcal{H}\right]=\mathrm{E}\left[L\left(h_{1}\right)^{p_{1}} \mid \mathcal{H}\right] \cdots \mathrm{E}\left[L\left(h_{n}\right)^{p_{n}} \mid \mathcal{H}\right]
$$

Lemma 7. Let $\mathcal{N}$ be a collection of $\mathrm{P}$-null events of $\mathcal{F}$. Then

$$
\mathcal{H} \subset \mathcal{F}_{L}=\sigma\{L(h): h \in H\} \vee \mathcal{N} .
$$

Proof. It is suffice to show that $\pi(C)$ is $\mathcal{F}_{L}$-measurable for all $C \in \mathcal{G}$.

Since the measure $M_{\pi}$ is $\sigma$-finite then there exists a sequence of pairwisedisjoint sets $A_{n} \times B_{n}, A_{n} \in \mathcal{G}$ and $B_{n} \in \mathcal{H}$ such that $\bigcup_{n=1}^{\infty} A_{n} \times B_{n}=T \times X \times \Omega$ and $M_{\pi}\left(A_{n} \times B_{n}\right)<\infty$. Then we have $M_{\pi}(C \times \Omega)=\sum_{n=1}^{\infty} M_{\pi}\left(\left(C \cap A_{n}\right) \times B_{n}\right)$ and $M_{\pi}\left(\left(C \cap A_{n}\right) \times B_{n}\right)<\infty$. Therefore $\pi\left(C \cap A_{n}\right) \mathbf{1}_{B_{n}}<\infty$ P-a.s. for all $n=1,2, \ldots$ and $\sum_{k=1}^{n} \pi\left(C \cap A_{k}\right) \mathbf{1}_{B_{k}} \rightarrow \pi(C)$ P-a.s. as $n \rightarrow \infty$. Hence it is suffice to show that $\pi\left(C \cap A_{k}\right) \mathbf{1}_{B_{k}}$ is $\mathcal{F}_{L}$-measurable.

Denote by $C_{k}^{m}$ the following sets $C_{k}^{m}=\left\{\pi\left(C \cap A_{k}\right) \leq m\right\}$. Then $C_{k}^{m} \in \mathcal{H}$, $\pi\left(C \cap A_{k}\right) \mathbf{1}_{C_{k}^{m}} \mathbf{1}_{B_{k}} \leq m$ and $\pi\left(C \cap A_{k}\right) \mathbf{1}_{C_{k}^{m}} \mathbf{1}_{B_{k}} \rightarrow \pi\left(C \cap A_{k}\right) \mathbf{1}_{B_{k}}$ P-a.s. as $m \rightarrow \infty$. Therefore the proof will be complete if we show that $\pi(U) \mathbf{1}_{D}$ is $\mathcal{F}_{L}$-measurable for all $U \in \mathcal{G}$ and $D \in \mathcal{H}$ such that $\pi(U) \mathbf{1}_{D}$ is bounded.

Let $U \in \mathcal{G}$ and $D \in \mathcal{H}$ be arbitrary sets such that $\pi(U) \mathbf{1}_{D} \leq Q$ a.s. For any $\omega \in D$ define measure $\pi^{\otimes 2}\left(d t_{1} d x_{1} d t_{2} d x_{2}, \omega\right)=\pi\left(d t_{1} d x_{1}, \omega\right) \pi\left(d t_{2} d x_{2}, \omega\right)$ on $\mathcal{G}^{\otimes 2}$. Since measures $\mu$ and $\nu$ without atoms then the measure $\pi$ has no atoms and $\pi^{\otimes 2}\left(\Delta_{2}^{U}, \omega\right)=0$, where $\Delta_{2}^{U}=\{(u, u): u \in U\}$. Therefore if we define measure $M_{\pi}^{2}(d z d \omega)=\pi^{\otimes 2}(d z, \omega) \mathrm{P}(d \omega)$ on $\mathcal{G}^{\otimes 2} \otimes \mathcal{H}$ then $M_{\pi}^{2}\left(\Delta_{2}^{U} \times D\right)=$ $\mathrm{E}\left(\pi^{\otimes 2}\left(\Delta_{2}^{U}\right) \mathbf{1}_{D}\right)=0$. Hence for any $m=1,2, \ldots$ there exists a system of sets $U_{k}^{m} \in \mathcal{G}$ and $D_{k}^{m} \in \mathcal{H}, k=1,2, \ldots$ such that $\bigcup_{k=1}^{\infty} U_{k}^{m} \times U_{k}^{m} \times D_{k}^{m} \supset \Delta_{2}^{U} \times D$, $\bigcup_{k=1}^{\infty} U_{k}^{m}=U, \bigcup_{k=1}^{\infty} D_{k}^{m}=D$ and $\sum_{k=1}^{\infty} \mathrm{E}\left(\pi\left(U_{k}^{m}\right)^{2} \mathbf{1}_{D_{k}^{m}}\right) \leq 1 / m$.

For any $n \geq 1$ we can find a system of pairwise-disjoint sets $\left\{V_{1}^{n}, \ldots, V_{p_{n}}^{n}\right\}$ $\subset \mathcal{G}$ and $\left\{E_{1}^{n}, \ldots, E_{q_{n}}^{n}\right\} \subset \mathcal{H}$, such that each $U_{k}^{m}$ and $D_{k}^{m} k=1, \ldots, n$ can be expressed as a disjoint union of some $V_{j}^{n}$ or $E_{j}^{n}$ respectively. Then we have

$$
\mathbf{1}_{\bigcup_{k=1}^{n} U_{k}^{m} \times U_{k}^{m} \times D_{k}^{m}}=\sum_{k=1}^{q_{n}} \sum_{j_{1}, j_{2}=1}^{p_{n}} \epsilon_{j_{1}, j_{2}, k}^{n} \mathbf{1}_{V_{j_{1}}^{n} \times V_{j_{2}}^{n} \times E_{k}^{n}},
$$


where $\epsilon_{j_{1}, j_{2}}^{n}$ is equal to 0 or 1 . Set

$$
\mathbf{1}_{Z_{n}}=\sum_{k=1}^{q_{n}} \sum_{j=1}^{p_{n}} \epsilon_{j, j, k}^{n} \mathbf{1}_{V_{j}^{n} \times E_{k}^{n}} .
$$

It is evident that $Z_{n} \subset U \times D$. Furthermore $\mathbf{1}_{Z_{n}}(s, \omega) \rightarrow \mathbf{1}_{U \times D}(s, \omega)$ as $n \rightarrow \infty$. Indeed, if $s \in U$ and $\omega \in D$ then $(s, s, \omega) \in \Delta_{2}^{U} \times D$ and there exists $n_{0} \geq 1$ such that $(s, s, \omega) \in \bigcup_{k=1}^{n} U_{k}^{m} \times U_{k}^{m} \times D_{k}^{m}$ for all $n \geq n_{0}$. Hence one can find $j_{n}$ and $k_{n}$ for all $n \geq n_{0}$ such that $\epsilon_{j_{n}, j_{n}, k_{n}}^{n}=1$. Therefore $Z_{n}(s, \omega)=1$ for all $n \geq n_{0}$.

It follows from dominated convergence theorem that

$$
\mathrm{E}\left(\sum_{k=1}^{q_{n}} \sum_{j=1}^{p_{n}} \epsilon_{j, j, k}^{n} \pi\left(V_{j}^{n}\right) \mathbf{1}_{E_{k}^{n}}-\pi(U) \mathbf{1}_{D}\right)^{2}=M_{\pi}\left(Z_{n}\right)-M_{\pi}(U \times D) \rightarrow 0 .
$$

Set $V_{0 j}^{n}=V_{j}^{n} \cap\left(T \times X_{0}\right)$ and let as calculate the following expectation:

$$
S_{n}=\mathrm{E}\left(\sum_{k=1}^{q_{n}} \sum_{j=1}^{p_{n}} \epsilon_{j, j, k}^{n}\left(L\left(\mathbf{1}_{V_{j}^{n}} \mathbf{1}_{E_{k}^{n}}\right)^{2}-L\left(\mathbf{1}_{V_{0 j}^{n}} \mathbf{1}_{E_{k}^{n}}\right)-\pi\left(V_{j}^{n}\right)\right) \mathbf{1}_{E_{k}^{n}}\right)^{2} .
$$

Since $\pi\left(V_{0 j}^{n}\right) \mathbf{1}_{E_{k}^{n}} \leq \pi\left(V_{j}^{n}\right) \mathbf{1}_{E_{k}^{n}} \leq \pi(U) \mathbf{1}_{D} \leq Q$ then $\mathbf{1}_{V_{j}^{n}} \mathbf{1}_{E_{k}^{n}} \in K$ and from Lemma 6 we get

$$
\begin{aligned}
& S_{n}=\sum_{k=1}^{q_{n}} \sum_{i, j=1}^{p_{n}} \epsilon_{j, j, k}^{n} \epsilon_{i, i, k}^{n} \mathrm{E}\left(\left(L\left(\mathbf{1}_{V_{i}^{n}} \mathbf{1}_{E_{k}^{n}}\right)^{2}-L\left(\mathbf{1}_{V_{0 i}^{n}} \mathbf{1}_{E_{k}^{n}}\right)-\pi\left(V_{i}^{n}\right)\right)\right. \\
& \left.\times\left(L\left(\mathbf{1}_{V_{j}^{n}} \mathbf{1}_{E_{k}^{n}}\right)^{2}-L\left(\mathbf{1}_{V_{0 j}^{n}} \mathbf{1}_{E_{k}^{n}}\right)-\pi\left(V_{j}^{n}\right)\right) \mathbf{1}_{E_{k}^{n}}\right) \\
& =\sum_{k=1}^{q_{n}} \sum_{i \neq j} \epsilon_{j, j, k}^{n} \epsilon_{i, i, k}^{n} \mathrm{E}\left(\left(\pi\left(V_{i}^{n}\right) \pi\left(V_{j}^{n}\right)-\pi\left(V_{i}^{n}\right) \pi\left(V_{j}^{n}\right)-\pi\left(V_{i}^{n}\right) \pi\left(V_{j}^{n}\right)\right.\right. \\
& \left.\left.+\pi\left(V_{i}^{n}\right) \pi\left(V_{j}^{n}\right)\right) \mathbf{1}_{E_{k}^{n}}\right)+\sum_{k=1}^{q_{n}} \sum_{j=1}^{p_{n}} \epsilon_{j, j, k}^{n} \mathrm{E}\left(\left(L\left(\mathbf{1}_{V_{j}^{n}} \mathbf{1}_{E_{k}^{n}}\right)^{4}+L\left(\mathbf{1}_{V_{0 j}^{n}} \mathbf{1}_{E_{k}^{n}}\right)^{2}+\pi\left(V_{j}^{n}\right)^{2}\right.\right. \\
& \left.\left.-2 L\left(\mathbf{1}_{V_{j}^{n}} \mathbf{1}_{E_{k}^{n}}\right)^{2} L\left(\mathbf{1}_{V_{0 j}^{n}} \mathbf{1}_{E_{k}^{n}}\right)-2 L\left(\mathbf{1}_{V_{j}^{n}} \mathbf{1}_{E_{k}^{n}}\right)^{2} \pi\left(V_{j}^{n}\right)\right) \mathbf{1}_{E_{k}^{n}}\right) .
\end{aligned}
$$

Taking respective derivatives at zero of conditional characteristic function of $L$ given by formula (1) yields $\mathrm{E}\left[L\left(\mathbf{1}_{V_{j}^{n}} \mathbf{1}_{E_{k}^{n}}\right)^{4} \mid \mathcal{H}\right]=\left(3 \pi\left(V_{j}^{n}\right)^{2}+\pi\left(V_{0 j}^{n}\right)\right) \mathbf{1}_{E_{k}^{n}}$ and $\mathrm{E}\left[L\left(\mathbf{1}_{V_{j}^{n}} \mathbf{1}_{E_{k}^{n}}\right)^{2} L\left(\mathbf{1}_{V_{0 j}^{n}} \mathbf{1}_{E_{k}^{n}}\right) \mid \mathcal{H}\right]=\pi\left(V_{0 j}^{n}\right) \mathbf{1}_{E_{k}^{n}}$. Therefore we have

$$
\begin{aligned}
S_{n}=\sum_{k=1}^{q_{n}} \sum_{j=1}^{p_{n}} \epsilon_{j, j, k}^{n} \mathrm{E}\left(\left(3 \pi\left(V_{j}^{n}\right)^{2}+\pi\left(V_{0 j}^{n}\right)+\pi\left(V_{0 j}^{n}\right)+\pi\left(V_{j}^{n}\right)^{2}\right.\right. \\
\left.\left.\quad-2 \pi\left(V_{0 j}^{n}\right)-2 \pi\left(V_{j}^{n}\right)^{2}\right) \mathbf{1}_{E_{k}^{n}}\right)=2 \mathrm{E}\left(\sum_{k=1}^{q_{n}} \sum_{j=1}^{p_{n}} \epsilon_{j, j, k}^{n} \pi\left(V_{j}^{n}\right)^{2} \mathbf{1}_{E_{k}^{n}}\right) .
\end{aligned}
$$


The last equality and formula (6) imply

$$
S_{n} \leq 2 \mathrm{E}\left(\sum_{k=1}^{q_{n}} \sum_{i, j=1}^{p_{n}} \epsilon_{i, j, k}^{n} \pi\left(V_{i}^{n}\right) \pi\left(V_{j}^{n}\right) \mathbf{1}_{E_{k}^{n}}\right) \leq 2 \sum_{k=1}^{\infty} \mathrm{E}\left(\pi\left(U_{k}^{m}\right)^{2} \mathbf{1}_{D_{k}^{m}}\right) \leq 2 / m .
$$

Therefore from expressions (7) and (8) we deduce that

$$
\sum_{k=1}^{q_{n}} \sum_{j=1}^{p_{n}} \epsilon_{j, j, k}^{n}\left(L\left(\mathbf{1}_{V_{j}^{n}} \mathbf{1}_{E_{k}^{n}}\right)^{2}-L\left(\mathbf{1}_{V_{0 j}^{n}} \mathbf{1}_{E_{k}^{n}}\right)\right) \mathbf{1}_{E_{k}^{n}} \rightarrow \pi(U) \mathbf{1}_{D}
$$

as $n, m \rightarrow \infty$. Hence $\pi(U) \mathbf{1}_{D}$ is $\mathcal{F}_{L}$ measurable, which completes the proof of the lemma.

In what follows we will always assume that $\mathcal{F}$ is a completion of $\mathcal{F}_{L}=$ $\sigma\{L(h), h \in H\}$.

Now we will introduce the generalized orthogonal polynomials $P_{n}$ (see, e.g. [33]). Denote by $\bar{x}=\left(x_{1}, x_{2}, \ldots, x_{n}, \ldots\right)$ a sequence of real numbers.

Define a function $F(z, \bar{x})$ by

$$
F(z, \bar{x})=\exp \left(\sum_{k=1}^{\infty}(-1)^{k+1} \frac{z^{k}}{k} x_{k}\right) .
$$

If $R(\bar{x})=\left(\limsup \left|x_{k}\right|^{1 / k}\right)^{-1}>0$ then the series in (9) converges for all $|z|<R(\bar{x})$. So the function $F(z, \bar{x})$ is analytic for $|z|<R(\bar{x})$.

Consider an expansion in powers of $z$ of the function $F(z, \bar{x})$

$$
F(z, \bar{x})=\sum_{n=0}^{\infty} z^{n} P_{n}(\bar{x})
$$

Using this development, one can easily show the following equalities:

$$
\begin{aligned}
(n+1) P_{n+1}(\bar{x}) & =\sum_{k=0}^{n}(-1)^{k} x_{k+1} P_{n-k}(\bar{x}), \quad n \geq 0, \\
\frac{\partial}{\partial x_{l}} P_{n}(\bar{x}) & = \begin{cases}0, & \text { if } l>n, \\
(-1)^{l+1} \frac{1}{l} P_{n-l}(\bar{x}), & \text { if } l \leq n .\end{cases}
\end{aligned}
$$

Indeed, (10) and (11) follow from $\frac{\partial F}{\partial z}=\sum_{k=0}^{\infty}(-1)^{k} z^{k} x_{k+1} F$, respectively, and $\frac{\partial F}{\partial x_{l}}=(-1)^{l+1} \frac{F}{l} z^{l}$. From (11) it follows that $P_{n}$ depends only on finite number of variables, namely $x_{1}, x_{2}, \ldots, x_{n}$. Since $P_{0} \equiv 1$, then (10) implies that $P_{n}\left(x_{1}, x_{2}, \ldots, x_{n}\right)$ is a polynomial with the highest order term $\frac{x_{1}^{n}}{n !}$. The first polynomials are $P_{1}\left(x_{1}\right)=x_{1}$ and $P_{2}\left(x_{1}, x_{2}\right)=\frac{1}{2}\left(x_{1}^{2}-x_{2}\right)$.

Using the equality $F(z, \bar{x}+\bar{y})=F(z, \bar{x}) F(z, \bar{y})$, where $\bar{y}=\left(y_{1}, y_{2}, \ldots, y_{n}\right.$, $\ldots)$ and $\bar{x}+\bar{y}=\left(x_{1}+y_{1}, x_{2}+y_{2}, \ldots, x_{n}+y_{n}, \ldots\right)$ it is easy to show that 


$$
P_{n}(\bar{x}+\bar{y})=\sum_{k=0}^{n} P_{k}(\bar{x}) P_{n-k}(\bar{y})
$$

If $\bar{u}(y)=\left(y, y^{2}, y^{3}, \ldots, y^{n}, \ldots\right)$ then $F(z, \bar{u}(y))=1+z y$ for $|z y|<1$. Hence $P_{1}(\bar{u}(y))=y$ and $P_{n}(\bar{u}(y))=0$ for all $n \geq 2$. Furthermore, equation (12) implies that

$$
P_{n}(\bar{x}+\bar{u}(y))-P_{n}(\bar{x})=y P_{n-1}(\bar{x}) .
$$

It is possible to find the explicit formula for polynomials $P_{n}$. Indeed, $P_{n}$ can be written in the following form:

$$
P_{n}\left(x_{1}, x_{2}, \ldots x_{n}\right)=\sum_{i_{1}+i_{2}+\cdots+i_{n} \leq n} a_{i_{1}, i_{2}, \ldots i_{n}} x_{1}^{i_{1}} x_{2}^{i_{2}} \cdots x_{n}^{i_{n}} .
$$

It is easy to see that

$$
\frac{\partial^{i_{1}+i_{2}+\cdots+i_{n}} P_{n}}{\partial x_{1}^{i_{1}} \partial x_{2}^{i_{2}} \cdots \partial x_{n}^{i_{n}}}(0, \ldots, 0)=i_{1} ! i_{2} ! \cdots i_{n} ! a_{i_{1}, i_{2}, \ldots i_{n}} .
$$

It follows from the equality (11) that

$$
\begin{gathered}
\frac{\partial^{i_{1}+i_{2}+\cdots+i_{n}} P_{n}}{\partial x_{1}^{i_{1}} \partial x_{2}^{i_{2}} \cdots \partial x_{n}^{i_{n}}}(0, \ldots, 0)= \\
\begin{cases}0, & \text { if } i_{1}+2 i_{2}+3 i_{3} \cdots+n i_{n} \neq n, \\
(-1)^{n+i_{1}+i_{2}+\cdots+i_{n}} 2^{-i_{2}} 3^{-i_{3}} \cdots n^{-i_{n}}, & \text { if } i_{1}+2 i_{2}+3 i_{3} \cdots+n i_{n}=n .\end{cases}
\end{gathered}
$$

Hence

$$
\begin{aligned}
& P_{n}\left(x_{1}, x_{2}, \ldots x_{n}\right)= \\
& \sum_{i_{1}+2 i_{2}+3 i_{3} \cdots+n i_{n}=n}(-1)^{n+i_{1}+i_{2}+\cdots+i_{n}} \frac{x_{1}^{i_{1}} x_{2}^{i_{2}} \cdots x_{n}^{i_{n}}}{i_{1} ! i_{2} ! \cdots i_{n} ! 2^{i_{2}} 3^{i_{3}} \cdots n^{i_{n}}} .
\end{aligned}
$$

For $h \in K$ let $\bar{x}(h)=\left(x_{1}(h), x_{2}(h), \ldots x_{n}(h), \ldots\right)$ denote the sequence of the random variables, such that $x_{1}(h)=L(h), x_{2}(h)=L\left(h^{2} \mathbf{1}_{X_{0}}\right)+$ $\int_{T \times X} h^{k}(t, x) \pi(d t d x)=L\left(h^{2} \mathbf{1}_{X_{0}}\right)+\pi\left(h^{2}\right), x_{k}(h)=L\left(h^{k} \mathbf{1}_{X_{0}}\right)+$ $\int_{T \times X_{0}} h^{k}(t, x) \nu(d t d x)=L\left(h^{k} \mathbf{1}_{X_{0}}\right)+\pi\left(h^{k} \mathbf{1}_{X_{0}}\right), k=3,4, \ldots$

The relationship between generalized orthogonal polynomials and conditional additive processes on $H$ is given by the following result.

Lemma 8. Let $h$ and $g \in K$. Then for all $n, m \geq 0$ we have $P_{n}(\bar{x}(h))$ and $P_{m}(\bar{x}(g)) \in L^{2}(\Omega)$, and

$$
\mathrm{E}\left[P_{n}(\bar{x}(h)) P_{m}(\bar{x}(g)) \mid \mathcal{H}\right]= \begin{cases}0, & \text { if } n \neq m \\ \frac{1}{n !}(\mathrm{E}[L(h) L(g) \mid \mathcal{H}])^{n}, & \text { if } n=m .\end{cases}
$$


Proof. Since $h, g \in K$ and $P_{n}, P_{m}$ are the polynomials, then by Lemma 6 we have $P_{n}(\bar{x}(h))$ and $P_{m}(\bar{x}(g)) \in L^{2}(\Omega, \mathcal{F}, \mathrm{P})$.

Denote by $\phi(z, \bar{x})$ the power of the exponent in the formula (9):

$$
\phi(z, \bar{x})=\sum_{k=1}^{\infty}(-1)^{k+1} \frac{z^{k}}{k} x_{k} .
$$

Since

$$
\begin{array}{r}
\frac{1}{R}=\limsup _{k \rightarrow \infty}\left\|x_{k}(h)\right\|_{L^{2}(\Omega)}^{1 / k}=\lim _{k \rightarrow \infty}\left[\mathrm{E}\left(L\left(h^{k} \mathbf{1}_{X_{0}}\right)^{2}\right)+\mathrm{E}\left(\pi\left(h^{k} \mathbf{1}_{X_{0}}\right)^{2}\right)\right]^{1 / 2 k} \\
\leq \lim _{k \rightarrow \infty}\left(\left\|h \mathbf{1}_{X_{0}}\right\|_{L^{\infty}}^{2 k-2} \mathrm{E}\left(\pi\left(h^{2} \mathbf{1}_{X_{0}}\right)\right)+\left\|h \mathbf{1}_{X_{0}}\right\|_{L^{\infty}}^{2 k-4} \mathrm{E}\left(\pi\left(h^{2} \mathbf{1}_{X_{0}}\right)^{2}\right)\right)^{1 / 2 k} \\
\leq\left\|h \mathbf{1}_{X_{0}}\right\|_{L^{\infty}} .
\end{array}
$$

Then the series

$$
\sum_{k=1}^{\infty} \frac{|z|^{k}}{k}\left\|x_{k}(h)\right\|_{L^{2}(\Omega)}
$$

converges if $|z|<1 /\left\|h \mathbf{1}_{X_{0}}\right\|_{L^{\infty}} \leq R$, which implies that $\phi(z, \bar{x}(h)) \in L^{2}(\Omega)$ for all $|z|<1 /\left\|h \mathbf{1}_{X_{0}}\right\|_{L^{\infty}}$.

Let's note that for all $|z|<1 /\left\|h \mathbf{1}_{X_{0}}\right\|_{L^{\infty}}$ we have $\ln \left(1+z h \mathbf{1}_{X_{0}}\right) \in H$. Indeed, by using Taylor's formula, we get

$$
\left(\ln \left(1+z h \mathbf{1}_{X_{0}}\right)\right)^{2} \leq \frac{z^{2} h^{2} \mathbf{1}_{X_{0}}}{\left(1-|z||| h \mathbf{1}_{X_{0}} \|_{L^{\infty}}\right)^{2}} .
$$

In the same way one can obtain the following inequality

$$
\left|\ln \left(1+z h \mathbf{1}_{X_{0}}\right)-z h \mathbf{1}_{X_{0}}\right| \leq \frac{z^{2} h^{2} \mathbf{1}_{X_{0}}}{2\left(1-\left.|z||| h \mathbf{1}_{X_{0}}\right|_{L^{\infty}}\right)^{2}},
$$

which implies that $\ln \left(1+z h(t, x) \mathbf{1}_{X_{0}}\right)-z h(t, x) \mathbf{1}_{X_{0}}$ is integrable with respect to measure $M_{\pi}$ for all $|z|<1 /\left\|h \mathbf{1}_{X_{0}}\right\|_{L^{\infty}}$.

So by using the linearity and the continuity of the mapping $h \rightarrow L(h)$ we have for all $|z|<1 /|| h \mathbf{1}_{X_{0}} \|_{L^{\infty}}$

$$
\begin{aligned}
\phi(z, \bar{x}(h))= & \sum_{k=2}^{\infty}(-1)^{k+1} \frac{z^{k}}{k}\left(L\left(h^{k} \mathbf{1}_{X_{0}}\right)+\pi\left(h^{k} \mathbf{1}_{X_{0}}\right)\right)+z L(h)-\frac{z^{2}}{2} \pi\left(h \mathbf{1}_{\Delta}\right) \\
& =L\left(\ln \left(1+z h \mathbf{1}_{X_{0}}\right)+z h \mathbf{1}_{\Delta}\right) \\
& +\int_{T \times X_{0}}(\ln (1+z h(t, x))-z h(t, x)) \nu(d t d x)-\frac{z^{2}}{2} \pi\left(h \mathbf{1}_{\Delta}\right) .
\end{aligned}
$$

We claim that $w=\ln \left(1+z h \mathbf{1}_{X_{0}}\right)+z h \mathbf{1}_{\Delta} \in K$ for all $|z|<1 /\left\|h \mathbf{1}_{X_{0}}\right\|_{L^{\infty}}$. Indeed 


$$
\left|w \mathbf{1}_{X_{0}}\right|=\left|\ln \left(1+z h \mathbf{1}_{X_{0}}\right)\right| \leq \frac{\left.|z||| h \mathbf{1}_{X_{0}}\right|_{L^{\infty}}}{|1-| z||\left|h \mathbf{1}_{X_{0}}\right|_{L^{\infty}} \mid}
$$

and since $h \in K$ then we have for some constant $C_{1}>0$

$$
\begin{aligned}
\pi\left(w^{2}\right)=z^{2} \pi\left(h^{2} \mathbf{1}_{\Delta}\right)+\pi\left(\ln ^{2}(\right. & \left.\left.+z h \mathbf{1}_{X_{0}}\right)\right) \\
& \leq z^{2} \pi\left(h^{2} \mathbf{1}_{\Delta}\right)+\frac{z^{2} \pi\left(h^{2} \mathbf{1}_{X_{0}}\right)}{\left(1-|z|\left\|h \mathbf{1}_{X_{0}}\right\|_{L^{\infty}}\right)^{2}} \leq C_{1} .
\end{aligned}
$$

Hence from inequality $\ln (1+x) \leq x$, equality (15) and Lemma 6 we have for all $|z|<1 /\left\|h \mathbf{1}_{X_{0}}\right\|_{L^{\infty}}$

$$
\begin{aligned}
\mathrm{E}\left(F(z, \bar{x}(h))^{2}\right)=\mathrm{E}[\exp (2 \phi(z, & \bar{x}(h)))] \\
& \leq \mathrm{E}\left[\exp \left(2 L\left(\ln \left(1+z h \mathbf{1}_{X_{0}}\right)+z h \mathbf{1}_{\Delta}\right)\right)\right]<\infty
\end{aligned}
$$

So $F(z, \bar{x}(h)) \in L^{2}(\Omega)$ if $|z|<1 /\left\|h \mathbf{1}_{X_{0}}\right\|_{L^{\infty}}$.

$$
\begin{aligned}
& \mathrm{E}[F(z, \bar{x}(h)) F(y, \bar{x}(g)) \mid \mathcal{H}]=\mathrm{E}[\exp (\phi(z, \bar{x}(h))+\phi(y, \bar{x}(g))) \mid \mathcal{H}] \\
& =\mathrm{E}\left[\operatorname { e x p } \left(L\left(\ln \left[\left(1+z h \mathbf{1}_{X_{0}}\right)\left(1+y g \mathbf{1}_{X_{0}}\right)\right]\right)\right.\right. \\
& +\int_{T \times X_{0}}(\ln [(1+z h(t, x))(1+y g(t, x))]-z h(t, x)-y g(t, x)) \nu(d t d x) \\
& \left.\left.+L\left(z h \mathbf{1}_{\Delta}+y g \mathbf{1}_{\Delta}\right)-\frac{1}{2} \int_{T}\left(z^{2} h^{2}(t, \Delta)+y^{2} g^{2}(t, \Delta)\right) \mu(d t)\right) \mid \mathcal{H}\right] \\
& =\exp \left(\int_{T \times X_{0}}\left(e^{\ln [(1+z h(t, x))(1+y g(t, x))]}-1-\ln [(1+z h(t, x))(1+y g(t, x))]\right) \nu(d t d x)\right. \\
& +\int_{T \times X_{0}}(\ln [(1+z h(t, x))(1+y g(t, x))]-z h(t, x)-y g(t, x)) \nu(d t d x) \\
& \left.+\frac{1}{2} \int_{T}\left((z h(t, \Delta)+y g(t, \Delta))^{2}-z^{2} h^{2}(t, \Delta)-y^{2} g^{2}(t, \Delta)\right) \mu(d t)\right) \\
& =\exp \left(z y \int_{T \times X} h(t, x) g(t, x) \pi(d t d x)\right)=\exp (z y \mathrm{E}[L(h) L(g) \mid \mathcal{H}]),
\end{aligned}
$$

where we have used Lemma 6 to calculate the conditional expectation.

Taking the $(n+m)$-th partial derivative $\frac{\partial^{n+m}}{\partial z^{n} \partial y^{m}}$ at $z=y=0$ in both sides of the above equality yields

$$
\mathrm{E}\left[n ! m ! P_{n}(\bar{x}(h)) P_{m}(\bar{x}(g)) \mid \mathcal{H}\right]= \begin{cases}0, & \text { if } n \neq m \\ n !(\mathrm{E}[L(h) L(g) \mid \mathcal{H}])^{n}, & \text { if } n=m\end{cases}
$$

Lemma 9. The random variables $\left\{e^{L(h)}, h \in K\right\}$ form a total subset of $L^{2}(\Omega, \mathcal{F}, P)$. 
Proof. It follows from Lemma 6 that $e^{L(h)} \in L^{2}(\Omega)$ if $h \in K$.

Let $\xi \in L^{2}(\Omega)$ be such that $\mathrm{E}\left(\xi e^{L(h)}\right)=0$ for all $h \in K$. The linearity of the mapping $h \rightarrow L(h)$ implies

$$
\mathrm{E}\left(\xi \exp \sum_{k=1}^{n} z_{k} L\left(h_{k}\right)\right)=0
$$

for any $z_{1}, \ldots, z_{n} \in \mathbb{R}, h_{1}, \ldots, h_{n} \in K, n \geq 1$. Suppose that $n \geq 1$ and $h_{1}, \ldots, h_{n} \in K$ are fixed. Then (16) says that Laplace transform of the signed measure

$$
\tau(B)=\mathrm{E}\left(\xi \mathbf{1}_{B}\left(L\left(h_{1}\right), \ldots, L\left(h_{n}\right)\right)\right),
$$

where $B$ is a Borel subset of $\mathbb{R}^{n}$, is identically zero on $\mathbb{R}^{n}$. Consequently, this measure is zero, which implies $\mathrm{E}\left(\xi \mathbf{1}_{G}\right)=0$ for any $G \in \mathcal{F}$. So $\xi=0$, completing the proof of the lemma.

For each $n \geq 0$ we will denote by $\mathcal{P}_{n}$ the closed linear subspace of $L^{2}(\Omega, \mathcal{F}, P)$ generated by the random variables $\left\{\xi P_{n}(\bar{x}(h)): h \in K, \xi \in\right.$ $\left.L^{\infty}(\Omega, \mathcal{H}, \mathrm{P})\right\} . \mathcal{P}_{0}$ will be the set $L^{2}(\Omega, \mathcal{H}, P)$ of $\mathcal{H}$-measurable square integrable random variables. For $n=1, \mathcal{P}_{1}$ coincides with the set of random variables $\{L(h): h \in H\}$. From Lemma 8 we obtain that $\mathcal{P}_{n}$ and $\mathcal{P}_{m}$ are orthogonal whenever $n \neq m$. We will call the space $\mathcal{P}_{n}$ chaos of order $n$.

Theorem 10. The space $L^{2}(\Omega, \mathcal{F}, P)$ can be decomposed into the infinite orthogonal sum of the subspaces $\mathcal{P}_{n}$ :

$$
L^{2}(\Omega, \mathcal{F}, P)=\bigoplus_{n=0}^{\infty} \mathcal{P}_{n} .
$$

Proof. Let $\xi \in L^{2}(\Omega, \mathcal{F}, P)$ such that $\xi$ is orthogonal to all $\mathcal{P}_{n}, n \geq 0$. We have to show that $\xi=0$. For all $h \in K$ and $\eta \in L^{\infty}(\Omega, \mathcal{H}, P)$ we get $\mathrm{E}\left(\xi \eta P_{n}(\bar{x}(h))\right)=0$. Hence $\mathrm{E}\left[\xi P_{n}(\bar{x}(h)) \mid \mathcal{H}\right]=0$. Since from the proof of Lemma 8 we have that $F(z, \bar{x}(h)) \in L^{2}(\Omega)$ for all $z<1 /\left\|h \mathbf{1}_{X_{0}}\right\|_{L^{\infty}}$, then $\mathrm{E}[\xi F(z, \bar{x}(h)) \mid \mathcal{H}]=0$ for $z<1 /\left\|h \mathbf{1}_{X_{0}}\right\|_{L^{\infty}}$. Using equality (15) we obtain

$$
\begin{array}{r}
0=\mathrm{E}[\xi F(z, \bar{x}(h)) \mid \mathcal{H}]=\mathrm{E}\left[\xi e^{\phi(z, \bar{x}(h))} \mid \mathcal{H}\right]=\mathrm{E}\left[\xi \operatorname { e x p } \left(L\left(\ln \left(1+z h \mathbf{1}_{X_{0}}\right)\right)\right.\right. \\
+\int_{T \times X_{0}}(\ln (1+z h(t, x))-z h(t, x)) \nu(d t d x)+L\left(z h \mathbf{1}_{\Delta}\right) \\
\left.\left.-\frac{1}{2} \int_{T} z^{2} h^{2}(t, \Delta) \mu(d t)\right) \mid \mathcal{H}\right]
\end{array}
$$

Thus for any $z<1 / \|\left. h \mathbf{1}_{X_{0}}\right|_{L^{\infty}}$

$$
\mathrm{E}\left[\xi \exp \left(L\left(\ln \left(1+z h \mathbf{1}_{X_{0}}\right)\right)+L\left(z h \mathbf{1}_{\Delta}\right)\right)\right]=0 .
$$

We claim that if $h \in K$ such that $h \geq \varepsilon-1 M_{\pi}$-a.e. for some $1 \geq$ $\varepsilon>0$ then equality (17) holds for $z=1$. Indeed the right-hand side of the 
expression (17) meaningful in this case for all $z \in[0 ; 1]$. The extension to the complex numbers $\operatorname{Re} z \in[0 ; 1]$ is evident. Denote this function by $\Phi(z)$. Since $\left|h \mathbf{1}_{X_{0}} /\left(1+z h \mathbf{1}_{X_{0}}\right)\right| \leq\left.|| h \mathbf{1}_{X_{0}}\right|_{L^{\infty}} / \varepsilon$ and $\pi\left(h^{2} \mathbf{1}_{X_{0}} /\left(1+z h \mathbf{1}_{X_{0}}\right)^{2}\right) \leq \pi\left(h^{2}\right) / \varepsilon^{2}$ then $h \mathbf{1}_{X_{0}} /\left(1+z h \mathbf{1}_{X_{0}}\right) \in K$ and the straightforward calculation shows that $\Phi(z)$ is differentiable and

$\Phi^{\prime}(z)=\mathrm{E}\left[\xi L\left(h \mathbf{1}_{X_{0}} /\left(1+z h \mathbf{1}_{X_{0}}\right)+h \mathbf{1}_{\Delta}\right) \exp \left(L\left(\ln \left(1+z h \mathbf{1}_{X_{0}}\right)\right)+L\left(z h \mathbf{1}_{\Delta}\right)\right)\right]$.

Hence $\Phi(z)$ is an analytical function for $\operatorname{Re} z \in[0 ; 1]$. Consequently the uniqueness theorem yields the desired statement.

For any $g \in K$ we have $\left(e^{g}-1\right) \in K$ and $\left(e^{g}-1\right) \mathbf{1}_{X_{0}}>-1+\varepsilon M_{\pi^{-}}$-a.e. for some $1 \geq \varepsilon>0$. Putting in (17) $h=\left(e^{g}-1\right) \mathbf{1}_{X_{0}}+g \mathbf{1}_{\Delta}$ and $z=1$ we deduce that $\mathrm{E}\left(\xi e^{L(g)}\right)=0$ for all $g \in K$. By Lemma 9 we get $\xi=0$, which completes the proof of the theorem.

\section{Multiple integrals}

The purpose of the section is to define multiple stochastic integrals with respect to $L$ and to show that the $n$th chaos $\mathcal{P}_{n}$ is generated by these multiple stochastic integrals. The construction of multiple stochastic integrals for processes with independent increments provided by Itô in [11]. For its generalization to other classes of processes the reader referred to $[9,13,21,32]$.

Recall that random measure $\pi(d t d x, \omega)$ has no atoms for all $\omega \in \Omega$. It means that neither measure $\mu$ nor measure $\nu$ has no atoms for all $\omega \in \Omega$. Since a separable Hilbert space $H$ has the form $H=L^{2}\left(T \times X \times \Omega, \mathcal{G} \otimes \mathcal{H}, M_{\pi}\right)$, where $M_{\pi}(d t d x d \omega)=\pi(d t d x, \omega) P(d \omega)$ is a $\sigma$-finite measure, then the process $L$ is characterized by the family of random variables $\left\{L(A), A \in \mathcal{G} \otimes \mathcal{H}, M_{\pi}(A)<\right.$ $\infty$, where $L(A)=L\left(\mathbf{1}_{A}\right)$. We can consider $L(A)$ as a $L^{2}(\Omega, \mathcal{F}, P)$-valued measure on the parametric space $(T \times X \times \Omega, \mathcal{G} \otimes \mathcal{H})$, which takes conditionally independent values on any family of disjoint subsets of $T \times X \times \Omega$.

Fix $m \geq 1$. Denote by $M_{\pi}^{m}$ the following measure

$$
M_{\pi}^{m}\left(d t_{1} d x_{1} \cdots d t_{m} d x_{m} d \omega\right)=\pi\left(d t_{1} d x_{1}, \omega\right) \cdots \pi\left(d t_{m} d x_{m}, \omega\right) \mathrm{P}(d \omega)
$$

defined on the $\sigma$-algebra $\mathcal{G}^{\otimes m} \otimes \mathcal{F}$. In this section will consider only the restriction of this measure on the $\sigma$-algebra $\mathcal{G}^{\otimes m} \otimes \mathcal{H}$. Since the measure $M_{\pi}$ is $\sigma$-finite then $M_{\pi}^{m}$ will be $\sigma$-finite. Indeed if the sequence of pairwise-disjoint sets $A_{n} \times B_{n}, A_{n} \in \mathcal{G}$ and $B_{n} \in \mathcal{H}$ such that $\bigcup_{n=1}^{\infty} A_{n} \times B_{n}=T \times X \times \Omega$ and $M_{\pi}\left(A_{n} \times B_{n}\right)<\infty$, then setting $B_{n}^{k}=\left\{k-1 \leq \pi\left(A_{n} \times B_{n}\right)<k\right\}$ we have $\bigcup_{k=1}^{\infty} A_{n} \times B_{n}^{k}=A_{n} \times B_{n}$. Denote $B_{n_{1} n_{2} \ldots n_{m}}^{k_{1} k_{2} \ldots k_{m}}=\bigcap_{j=1}^{m} B_{n_{j}}^{k_{j}}$ then $M_{\pi}^{m}\left(A_{n_{1}} \times A_{n_{2}} \times \cdots A_{n_{m}} \times B_{n_{1} n_{2} \ldots n_{m}}^{k_{1} k_{2} \ldots k_{m}}\right) \leq k_{1} k_{2} \cdots k_{m}<\infty$ and $(T \times X)^{m} \times \Omega=$ $\bigcup_{n_{1}, n_{2}, \ldots, n_{m}=1}^{\infty} \bigcup_{k_{1}, k_{2}, \ldots, k_{m}=1}^{\infty} A_{n_{1}} \times A_{n_{2}} \times \cdots A_{n_{m}} \times B_{n_{1} n_{2} \ldots n_{m}}^{k_{1} k_{2} \ldots k_{m}}$.

For any $\omega \in \Omega$ we can define measure $\pi^{\otimes m}\left(d t_{1} d x_{1} \cdots d t_{m} d x_{m}, \omega\right)$ on the $\sigma$ algebra $\mathcal{G}^{\otimes m}$ as a $m$-th power of the measure $\pi$. Since measure $\pi$ is $\sigma$-finite and 
without atoms then measure $\pi^{\otimes m}$ is $\sigma$-finite and without atoms. Moreover, $\pi^{\otimes m}\left(\Delta_{m}, \omega\right)=0$ for all $\omega \in \Omega$, where $\Delta_{m}=\left\{\left(t_{1}, \ldots, t_{m}\right): \exists t_{i}=t_{j}, i \neq j\right\}$ is a 'diagonal' set. Indeed, for fixed $\omega \in \Omega \sigma$-finiteness of $\pi$ implies that $T \times X=$ $\bigcup_{i=1}^{\infty} T_{i}$, where $T_{1}, T_{2}, \ldots$ are pairwise-disjoint sets in $\mathcal{G}$ and $\pi\left(T_{i}\right)<\infty$. Then $(T \times X)^{m}=\bigcup_{i_{1}, \ldots, i_{m}=1}^{\infty} T_{i_{1}} \times \cdots \times T_{i_{m}}$ and $\pi^{\otimes m}\left(T_{i_{1}} \times \cdots \times T_{i_{m}}\right)<\infty$. Define $C_{i_{1}, \ldots, i_{m}}=\left(T_{i_{1}} \times \cdots \times T_{i_{m}}\right) \bigcap \Delta_{m}$. Then $\Delta_{m}=\bigcup_{i_{1}, \ldots, i_{m}=1}^{\infty} C_{i_{1}, \ldots, i_{m}}$. It is easy to see that $C_{i_{1}, \ldots, i_{m}}=\emptyset$ if all the indices $i_{1}, \ldots, i_{m}$ are different. Hence it is enough to prove that $\pi^{\otimes m}\left(C_{i_{1}, \ldots, i_{m}}\right)=0$ if some of indices $i_{1}, \ldots, i_{m}$ are equal. Suppose that $i_{1}=i_{2}$. Using the nonexistence of atoms for the measure $\pi$ for any $n \in \mathbb{N}$ we can determine a system of pairwise-disjoints sets $\left\{V_{1}, \ldots, V_{n}\right\} \subset \mathcal{G}$, such that $\bigcup_{i=1}^{n} V_{i}=T_{i_{1}}$ and $\pi\left(V_{i}\right)=\pi\left(T_{i_{1}}\right) / n$ for every $i=$ $1, \ldots, n$. Then $C_{i_{1}, \ldots, i_{m}} \subset \bigcup_{i=1}^{n} V_{i} \times V_{i} \times T_{i_{3}} \times \cdots \times T_{i_{m}}$. Hence $\pi^{\otimes m}\left(C_{i_{1}, \ldots, i_{m}}\right) \leq$ $\sum_{i=1}^{n} \pi\left(V_{i}\right)^{2} \pi\left(T_{i_{3}}\right) \cdots \pi\left(T_{i_{m}}\right)=\pi\left(T_{i_{1}}\right)^{2} \pi\left(T_{i_{2}}\right) \pi\left(T_{i_{3}}\right) \cdots \pi\left(T_{i_{m}}\right) / n$. Letting $n$ tend to $\infty$ we obtain the desired result.

Precisely speaking the set $\Delta_{m}$ may not be an element of the $\sigma$-algebra $\mathcal{G}^{\otimes m}$ but the calculations above show that $\Delta_{m}$ belongs to completion $\overline{\mathcal{G}^{\otimes m}}{ }^{\omega}$ of $\mathcal{G}^{\otimes m}$ with respect to the measure $\pi^{\otimes m}(\cdot, \omega)$ for all $\omega \in \Omega$. Since $\pi^{\otimes m}(\cdot, \omega)$ can be extended to the $\sigma$-algebra $\overline{\mathcal{G}^{\otimes m}}=\bigcap_{\omega \in \Omega} \overline{\mathcal{G}^{\otimes m}}{ }^{\omega}$ and $\Delta_{m} \in \overline{\mathcal{G}^{\otimes m}}$ then measure $M_{\pi}^{m}$ can be extended on the $\sigma$-algebra $\overline{\mathcal{G}^{\otimes m}} \otimes \mathcal{H}$ and $M_{\pi}^{m}\left(\Delta_{m} \times \Omega\right)=0$. This fact is very important for definition of the multiple stochastic integral.

Set $\mathcal{G}_{0}=\left\{A \in \mathcal{G} \otimes \mathcal{H}: \mathbf{1}_{A} \in K\right\}$. We will define the multiple stochastic integral $I_{m}(f)$ of a function $f \in L^{2}\left((T \times X)^{m} \times \Omega, \mathcal{G}^{\otimes m} \otimes \mathcal{H}, M_{\pi}^{m}\right)$. Denote by $\mathcal{E}_{m}$ the set of elementary functions of the form

$$
\begin{aligned}
& f\left(t_{1}, x_{1}, \ldots, t_{m}, x_{m}, \omega\right)= \\
& \sum_{i_{1}, \ldots, i_{m}=1}^{n} a_{i_{1}, \ldots, i_{m}}(\omega) \mathbf{1}_{A_{i_{1}}}\left(t_{1}, x_{1}, \omega\right) \cdots \mathbf{1}_{A_{i_{m}}}\left(t_{m}, x_{m}, \omega\right),
\end{aligned}
$$

where $A_{1}, \ldots, A_{n}$ are pairwise-disjoint sets in $\mathcal{G}_{0}$, and the coefficients $a_{i_{1}, \ldots, i_{m}} \in$ $L^{\infty}(\Omega, \mathcal{H}, \mathrm{P})$ are zero if any two of indices $i_{1}, \ldots, i_{m}$ are equal.

For a function of the form (18) we define the multiple integral of the $m$-th order

$$
I_{m}(f)=\sum_{i_{1}, \ldots, i_{m}=1}^{n} a_{i_{1}, \ldots, i_{m}} L\left(A_{i_{1}}\right) \cdots L\left(A_{i_{m}}\right) .
$$

The definition does not depend on particular representation of $f$, and the following properties hold:

(i) $I_{m}(\alpha f+\beta g)=\alpha I_{m}(f)+\beta I_{m}(g)$ for all $\alpha$ and $\beta \in L^{\infty}(\Omega, \mathcal{H}, \mathrm{P}), f$ and $g$ in $\mathcal{E}_{m}$.

(ii) $I_{m}(f)=I_{m}(\widetilde{f})$, where $\widetilde{f}$ denotes the symmetrization of $f$ with respect to pairs of nonrandom variables, which is defined by

$$
\widetilde{f}\left(t_{1}, x_{1}, \ldots, t_{m}, x_{m}, \omega\right)=\frac{1}{m !} \sum_{\sigma} f\left(t_{\sigma(1)}, x_{\sigma(1)}, \ldots, t_{\sigma(m)}, x_{\sigma(m)}, \omega\right),
$$


(iii)

$\sigma$ running over all permutations of $\{1, \ldots, m\}$.

$$
\mathrm{E}\left[I_{m}(f) I_{p}(g) \mid \mathcal{H}\right]= \begin{cases}0, & \text { if } p \neq m \\ m ! \pi(\tilde{f} \widetilde{g}), & \text { if } p=m .\end{cases}
$$

The properties can be proved using Lemma 6 and exactly the same arguments as those used, for example, in [22, p. 8-9].

In order to extend the multiple stochastic integral to the space $L^{2}\left(M_{\pi}^{m}\right)$ we have to prove the following lemma.

Lemma 11. The space $\mathcal{E}_{m}$ is dense in $L^{2}\left((T \times X)^{m} \times \Omega, \mathcal{G}^{\otimes m} \otimes \mathcal{H}, M_{\pi}^{m}\right)$.

Proof. In order to show that $\mathcal{E}_{m}$ is dense in $L^{2}\left(M_{\pi}^{m}\right)$ it is suffices to show that the indicator function of any set $A \times B=A_{1} \times A_{2} \times \cdots \times A_{m} \times B$, where $A_{1}, \ldots, A_{m} \in \mathcal{G}, B \in \mathcal{H}$ and $M_{\pi}^{m}(A \times B)<\infty$ can be approximated by elementary functions in $\mathcal{E}_{m}$.

Denote by $B_{k}^{\prime}$ the following set

$$
B_{k}^{\prime}=\left\{\omega \in \Omega: \pi\left(A_{1}, \omega\right) \pi\left(A_{2}, \omega\right) \cdots \pi\left(A_{m}, \omega\right) \leq k\right\}
$$

. Since $M_{\pi}^{m}(A \times B)<\infty$ then $\mathbf{1}_{A_{1} \times \cdots \times A_{m} \times B_{k}} \rightarrow \mathbf{1}_{A \times B}$ as $k \rightarrow \infty$. Hence it is possible to assume that $\pi\left(A_{1}, \omega\right) \pi\left(A_{2}, \omega\right) \cdots \pi\left(A_{m}, \omega\right) \mathbf{1}_{B} \leq C$ a.s. for some positive constant $C$. This implies that $A_{i} \times B \in \mathcal{G}_{0}, i=1, \ldots, m$. Furthermore, it is possible to suppose that any sets $A_{i}$ and $A_{j}$ either equal or disjoint. Indeed, there exists a finite system of pairwise disjoint sets $\left\{A_{1}^{\prime}, \ldots, A_{n}^{\prime}\right\} \subset \mathcal{G}$ such that each $A_{i}$ can be expressed as a disjoint union of some of $A_{j}^{\prime}$. Then the indicator function of the set $A \times B$ can be represent as a finite sum of the indicator functions of the sets $A_{i_{1}}^{\prime} \times A_{i_{2}}^{\prime} \times \cdots \times A_{i_{m}}^{\prime} \times B$. If all indices $i_{1}, \ldots, i_{m}$ are different then it is an element of $\mathcal{E}_{m}$. For other indices some of the sets $A_{i_{k}}^{\prime}$ are equal.

Since $M_{\pi}^{m}\left(\Delta_{m} \times \Omega\right)=0$ then $M_{\pi}^{m}\left((A \times B) \bigcap\left(\Delta_{m} \times \Omega\right)\right)=0$ and for any $\epsilon>0$ there exists a system of sets $U_{k}^{\epsilon}=A_{1}^{k} \times \cdots A_{m}^{k} \times B_{k}, k=1,2, \ldots$ such that $\bigcup_{k=1}^{\infty} U_{k}^{\epsilon} \supset\left((A \times B) \bigcap\left(\Delta_{m} \times \Omega\right)\right), \sum_{k=1}^{\infty} M_{\pi}^{m}\left(U_{k}^{\epsilon}\right)<\epsilon, \bigcup_{k=1}^{\infty} A_{i}^{k}=A_{i}$, $i=1, \ldots, m$ and $\bigcup_{k=1}^{\infty} B_{k}=B$. Therefore $A_{i}^{k} \times B_{k} \in \mathcal{G}_{0}, i=1, \ldots, m$, $k=1,2, \ldots$ and $\bigcup_{k=1}^{\infty} U_{k}^{\epsilon} \subset A \times B$.

For any $n \geq 1$ we can find a system of pairwise disjoint sets $\left\{C_{1}^{n}, C_{2}^{n}, \ldots, C_{p_{n}}^{n}\right\} \subset \mathcal{G}$ and $\left\{B_{1}^{n}, B_{2}^{n}, \ldots, B_{q_{n}}^{n}\right\} \subset \mathcal{G}$, such that each $A_{i}^{k}$ and $B_{k}, i=1, \ldots, m, k=1, \ldots, n$ can be expressed as a disjoint union of some of $C_{j}^{n}$ or $B_{j}^{n}$ respectively. Notice that $C_{j}^{n} \times B_{i}^{n} \in \mathcal{G}_{0}$ for all $j=1, \ldots, p_{n}$, $i=1, \ldots, q_{n}$. We have

$$
\mathbf{1}_{\bigcup_{k=1}^{n} U_{k}^{\epsilon}}=\sum_{k=1}^{q_{n}} \sum_{j_{1}, \ldots, j_{m}=1}^{p_{n}} \epsilon_{j_{1}, \ldots, j_{m}, k}^{n} \mathbf{1}_{C_{j_{1}}^{n} \times C_{j_{2}}^{n} \times \cdots C_{j_{m}}^{n} \times B_{k}^{n}},
$$

where $\epsilon_{j_{1}, \ldots, j_{m}, k}^{n}$ is 0 or 1 . Let $J_{n}$ be the set of $m$-tuples $\left(j_{1}, \ldots, j_{m}\right), j_{i} \in$ $\left\{1,2, \ldots, p_{n}\right\}, i=1, \ldots, m$, where all the indices are different. We set 


$$
\mathbf{1}_{V_{n}^{\varepsilon}}=\sum_{k=1}^{q_{n}} \sum_{\left(j_{1}, \ldots, j_{m}\right) \in J_{n}}\left(1-\epsilon_{j_{1}, \ldots, j_{m}, k}^{n}\right) \mathbf{1}_{C_{j_{1}}^{n} \times C_{j_{2}}^{n} \times \cdots C_{j_{m}}^{n} \times B_{k}^{n}} .
$$

Then $\mathbf{1}_{V_{n}^{\varepsilon}}$ belongs to $\mathcal{E}_{m}$ and for $M_{\pi}^{m}$-a.e. $(t, \omega) \in\left(A \backslash \Delta_{m}\right) \times B$ we have $\mathbf{1}_{V_{n}^{\varepsilon}}(t, \omega)=1$ for all $\varepsilon \geq \varepsilon_{0}$ and $n \geq n_{0}$. Hence $\mathbf{1}_{V_{n}^{\varepsilon}} \rightarrow \mathbf{1}_{\left(A \backslash \Delta_{m}\right) \times B} M_{\pi}^{m}$-a.e. and in $L^{2}\left(M_{\pi}^{m}\right)$ as $n \rightarrow \infty$ and $\epsilon \rightarrow 0$. Finally the fact $M_{\pi}^{m}\left(\Delta_{m} \times \Omega\right)=0$ implies the proof of the lemma.

Letting $f=g$ in property (iii) obtains

$$
\mathrm{E}\left(I_{n}(f)^{2}\right)=m !\|\tilde{f}\|_{L^{2}\left(M_{\pi}^{m}\right)}^{2} \leq m !\|f\|_{L^{2}\left(M_{\pi}^{m}\right)}^{2} .
$$

Therefore, the operator $I_{m}$ can be extended to a linear and continuous operator from $L^{2}\left(M_{\pi}^{m}\right)$ to $L^{2}(\Omega, \mathcal{F}, \mathrm{P})$, which satisfies properties (i), (ii) and (iii).

If $f \in L^{2}\left(M_{\pi}^{p}\right)$ is symmetric function and $g \in K$ the contraction of one index of $f$ and $g$ is denoted by $f \otimes_{1} g$ and is defined by

$$
\begin{gathered}
\left(f \otimes_{1} g\right)\left(t_{1}, x_{1}, \ldots, t_{p-1}, x_{p-1}, \omega\right) \\
=\int_{T \times X} f\left(t_{1}, x_{1}, \ldots, t_{p-1}, x_{p-1}, s, z, \omega\right) g(s, z, \omega) \pi(d s d z, \omega) .
\end{gathered}
$$

The tensor product $f \otimes g$ will be understood as tensor product with respect to nonrandom variables, i.e.

$$
(f \otimes g)\left(t_{1}, x_{1}, \ldots, t_{p+1}, x_{p+1}, \omega\right)=f\left(t_{1}, x_{1}, \ldots, t_{p}, x_{p}, \omega\right) g\left(t_{p+1}, x_{p+1}, \omega\right) .
$$

Notice that $f \otimes_{1} g \in L^{2}\left(M_{\pi}^{p-1}\right)$ and $f \otimes g \in L^{2}\left(M_{\pi}^{p+1}\right)$ if $g \in K$.

The tensor product $f \otimes g$ and the contractions $f \otimes_{1} g$ are not necessarily symmetric. We will denote their symmetrization by $f \widetilde{\otimes} g$ and $f \widetilde{\otimes}_{1} g$ respectively.

The following, so called product formula, will be useful in the sequel. It was initially derived by Itô [10] for Gaussian case and by Kabanov [13] for Poisson case, then extended by Russo and Vallois [29] to products of two multiple stochastic integrals with respect to a normal martingale.

Proposition 12. Let $f \in L^{2}\left(M_{\pi}^{p}\right)$ be a symmetric function and let $g \in K$. Then

$$
I_{p}(f) I_{1}(g)=I_{p+1}(f \otimes g)+p I_{p-1}\left(f \otimes_{1} g\right)+p I_{p}\left(f g \mathbf{1}_{X_{0}}\right) .
$$

Proof. Since $g \in K$ then $f g \mathbf{1}_{X_{0}} \in L^{2}\left(M_{\pi}^{p}\right)$ and the right-hand side is correctly defined.

By the density of elementary functions in $L^{2}\left(M_{\pi}^{p}\right)$ and by properties (i) and (ii) we can assume that $f$ is the symmetrization of the function $\mathbf{1}_{A_{1}}\left(t_{1}, x_{1}, \omega\right) \mathbf{1}_{A_{2}}\left(t_{2}, x_{2}, \omega\right) \cdots \mathbf{1}_{A_{p}}\left(t_{p}, x_{p}, \omega\right)$, where the $A_{i}$ are pairwise-disjoint sets of $\mathcal{G}_{0}$, and $g=\mathbf{1}_{A_{1}}$ or $\mathbf{1}_{A_{0}}$, where $A_{0} \in \mathcal{G}_{0}$ is disjoint with $A_{1}, \ldots, A_{p}$. 
The case $g=\mathbf{1}_{A_{0}}$ is immediate because $f \otimes_{1} g=f g=0$ and $f \otimes g \in \mathcal{E}_{p+1}$. So, we assume $g=\mathbf{1}_{A_{1}}$. Since $A_{i} \in \mathcal{G}_{0}$ then $\pi\left(A_{1}\right) \pi\left(A_{2}\right) \cdots \pi\left(A_{p}\right) \leq C$ for some real constant $C>0$. Given $\epsilon>0$, according to Lemma 11 we can find the function $v_{\epsilon}^{\prime} \in \mathcal{E}_{2}$ such that $\left\|v_{\epsilon}^{\prime}-\mathbf{1}_{A_{1}} \otimes \mathbf{1}_{A_{1}}\right\|_{L^{2}\left(M_{\pi}^{2}\right)} \leq \epsilon$. It is possible to write $v_{\epsilon}^{\prime}$ in the following form

$$
v_{\epsilon}^{\prime}\left(t_{1}, x_{1}, t_{2}, x_{2}, \omega\right)=\sum_{i, j=1}^{k_{\epsilon}} a_{i j}^{\epsilon}(\omega) \mathbf{1}_{C_{i}}\left(t_{1}, x_{1}, \omega\right) \mathbf{1}_{C_{j}}\left(t_{2}, x_{2}, \omega\right),
$$

where $C_{1}, \ldots, C_{k_{\epsilon}}$ are pairwise disjoint subsets of $A_{1}$ in $\mathcal{G}_{0}, a_{i j}^{\epsilon} \in L^{\infty}(\Omega, \mathcal{H}, \mathrm{P})$ and $a_{i i}^{\epsilon}=0, i, j=1, \ldots, k_{\epsilon}$. Set $A_{1}^{0}=A_{1} \cap\left(T \times X_{0} \times \Omega\right)$ and

$$
\begin{aligned}
v_{\epsilon}\left(t_{1}, x_{1}, \ldots, t_{p+1},\right. & \left.x_{p+1}, \omega\right) \\
& =v_{\epsilon}^{\prime}\left(t_{1}, x_{1}, t_{2}, x_{2}, \omega\right) \mathbf{1}_{A_{2}}\left(t_{3}, x_{3}, \omega\right) \cdots \mathbf{1}_{A_{p}}\left(t_{p+1}, x_{p+1}, \omega\right) .
\end{aligned}
$$

Then $v_{\epsilon}$ is an elementary function and we have

$$
\begin{gathered}
I_{p}(f) I_{1}(g)=L\left(A_{1}\right)^{2} L\left(A_{2}\right) \cdots L\left(A_{p}\right) \\
=I_{p+1}\left(v_{\epsilon}\right)+\pi\left(A_{1}\right) L\left(A_{2}\right) \cdots L\left(A_{p}\right)+L\left(A_{1}^{0}\right) L\left(A_{2}\right) \cdots L\left(A_{p}\right) \\
+\left[\left(L\left(A_{1}\right)^{2}-\pi\left(A_{1}\right)-L\left(A_{1}^{0}\right)\right) L\left(A_{2}\right) \cdots L\left(A_{p}\right)-I_{p+1}\left(v_{\epsilon}\right)\right] \\
=I_{p+1}\left(v_{\epsilon}\right)+p I_{p-1}\left(f \otimes_{1} g\right)+p I_{p}\left(f g \mathbf{1}_{X_{0}}\right)+R_{\epsilon} .
\end{gathered}
$$

Indeed

$$
f \otimes_{1} g=\frac{1}{p} \pi\left(A_{1}\right) \operatorname{symm}\left(\mathbf{1}_{A_{2}} \otimes \cdots \otimes \mathbf{1}_{A_{p}}\right),
$$

and

$$
f g \mathbf{1}_{X_{0}}=\frac{1}{p} \mathbf{1}_{A_{1}^{0}} \otimes \operatorname{symm}\left(\mathbf{1}_{A_{2}} \otimes \cdots \otimes \mathbf{1}_{A_{p}}\right),
$$

where $\operatorname{symm}(\cdot)$ denotes the symmetrization with respect to the pairs of nonrandom variables of the function in parentheses. We have

$$
\begin{aligned}
& \left\|\tilde{v}_{\epsilon}-f \widetilde{\otimes} g\right\|_{L^{2}\left(M_{\pi}^{p+1}\right)}^{2}= \\
& \left\|\tilde{v}_{\epsilon}-\operatorname{symm}\left(\mathbf{1}_{A_{1}} \otimes \mathbf{1}_{A_{1}} \otimes \mathbf{1}_{A_{2}} \otimes \cdots \otimes \mathbf{1}_{A_{p}}\right)\right\|_{L^{2}\left(M_{\pi}^{p+1}\right)}^{2} \\
& \leq\left\|v_{\epsilon}-\mathbf{1}_{A_{1}} \otimes \mathbf{1}_{A_{1}} \otimes \mathbf{1}_{A_{2}} \otimes \cdots \otimes \mathbf{1}_{A_{p}}\right\|_{L^{2}\left(M_{\pi}^{p+1}\right)}^{2} \\
& =\mathrm{E}\left[\pi ( A _ { 2 } ) \cdots \pi ( A _ { p } ) \int _ { ( T \times X ) ^ { 2 } } \left(v_{\epsilon}^{\prime}\left(t_{1}, x_{1}, t_{2}, x_{2}\right)\right.\right. \\
& \left.\left.-\mathbf{1}_{A_{1}}\left(t_{1}, x_{1}\right) \mathbf{1}_{A_{1}}\left(t_{2}, x_{2}\right)\right)^{2} \pi\left(d t_{1} d x_{1}\right) \pi\left(d t_{2} d x_{2}\right)\right] \\
& \leq C\left\|v_{\epsilon}^{\prime}-\mathbf{1}_{A_{1}} \otimes \mathbf{1}_{A_{1}}\right\|_{L^{2}\left(M_{\pi}^{2}\right)}^{2} \leq C \epsilon^{2},
\end{aligned}
$$

and 


$$
\mathrm{E}\left(R_{\epsilon}^{2}\right)=\mathrm{E}\left(\left[L\left(A_{1}\right)^{2}-\pi\left(A_{1}\right)-L\left(A_{1}^{0}\right)-I_{2}\left(v_{\epsilon}^{\prime}\right)\right]^{2} L\left(A_{2}\right)^{2} L\left(A_{3}\right)^{2} \cdots L\left(A_{p}\right)^{2}\right) .
$$

Lemma 6 and properties of multiple integral imply

$$
\begin{gathered}
\mathrm{E}\left(R_{\epsilon}^{2}\right)=\mathrm{E}\left(\left[L\left(A_{1}\right)^{4}+\pi\left(A_{1}\right)^{2}+\pi\left(A_{1}^{0}\right)+2 \pi^{\otimes 2}\left({\widetilde{v^{\prime}}}_{\epsilon}^{2}\right)\right.\right. \\
\left.\left.-2 \pi\left(A_{1}\right)^{2}-2 L\left(A_{1}\right)^{2} L\left(A_{1}^{0}\right)-2 L\left(A_{1}\right)^{2} I_{2}\left(v_{\epsilon}^{\prime}\right)\right] \pi\left(A_{2}\right) \cdots \pi\left(A_{p}\right)\right) .
\end{gathered}
$$

Taking fourth derivative at $z=0$ of conditional characteristic function of $L$ given by formula (1) yields

$$
\mathrm{E}\left[L\left(A_{1}\right)^{4} \mid \mathcal{H}\right]=3 \pi\left(A_{1}\right)^{2}+\pi\left(A_{1}^{0}\right) .
$$

By using the same arguments since $A_{1}=A_{1}^{0} \cup\left(A_{1} \backslash A_{1}^{0}\right)$ we get

$$
\begin{aligned}
& \mathrm{E}\left[L\left(A_{1}\right)^{2} L\left(A_{1}^{0}\right) \mid \mathcal{H}\right]= \\
& \quad \mathrm{E}\left[L\left(A_{1}^{0}\right)^{3}+2 L\left(A_{1} \backslash A_{1}^{0}\right) L\left(A_{1}^{0}\right)^{2}+L\left(A_{1} \backslash A_{1}^{0}\right)^{2} L\left(A_{1}^{0}\right) \mid \mathcal{H}\right]=\pi\left(A_{1}^{0}\right) .
\end{aligned}
$$

It follows from equality (20) and Lemma 6 that

$$
\begin{gathered}
\mathrm{E}\left[L\left(A_{1}\right)^{2} I_{2}\left(v_{\epsilon}^{\prime}\right) \mid \mathcal{H}\right]=\sum_{i, j=1}^{k_{\epsilon}} a_{i j}^{\epsilon} \mathrm{E}\left[L\left(A_{1}\right)^{2} L\left(C_{i}\right) L\left(C_{j}\right) \mid \mathcal{H}\right] \\
=\sum_{i, j=1}^{k_{\epsilon}} a_{i j}^{\epsilon} \mathrm{E}\left[2 L\left(C_{i}\right)^{2} L\left(C_{j}\right)^{2} \mid \mathcal{H}\right]=2 \sum_{i, j=1}^{k_{\epsilon}} a_{i j}^{\epsilon} \pi\left(C_{i}\right) \pi\left(C_{j}\right)= \\
2 \int_{(T \times X)^{2}}{\widetilde{v^{\prime}}}_{\epsilon} d \pi^{\otimes 2}=2 \pi^{\otimes 2}\left({\widetilde{v^{\prime}}}_{\epsilon}\right) .
\end{gathered}
$$

Substituting expressions (24), (25) and (26) into (23) we have

$$
\begin{aligned}
\mathrm{E}\left(R_{\epsilon}^{2}\right)=\mathrm{E}\left[\left(2 \pi\left(A_{1}\right)^{2}+2 \pi^{\otimes 2}\left({\widetilde{v^{\prime}}}_{\epsilon}^{2}\right)-4 \pi^{\otimes 2}\left(\widetilde{v_{\epsilon}^{\prime}}\right)\right) \pi\left(A_{2}\right) \cdots \pi\left(A_{p}\right)\right] \\
=2 \mathrm{E}\left[\pi^{\otimes 2}\left(\left(\widetilde{v^{\prime}}{ }_{\epsilon}-\mathbf{1}_{A_{1}} \otimes \mathbf{1}_{A_{1}}\right)^{2}\right) \pi\left(A_{2}\right) \cdots \pi\left(A_{p}\right)\right] \\
\leq 2 C\left\|v_{\epsilon}^{\prime}-\mathbf{1}_{A_{1}} \otimes \mathbf{1}_{A_{1}}\right\|_{L^{2}\left(M_{\pi}^{2}\right)}^{2} \leq 2 C \epsilon^{2} .
\end{aligned}
$$

From formulae (21), (22) and (27) we obtain the desired result.

The next result gives the relationship between generalized orthogonal polynomials and multiple stochastic integrals.

Theorem 13. Let $P_{n}$ be the nth generalized orthogonal polynomial, and $\bar{x}(h)=\left(x_{k}(h)\right)_{k=1}^{\infty}$, where $x_{1}(h)=L(h), x_{2}(h)=L\left(h^{2} \mathbf{1}_{X_{0}}\right)+\|h\|_{H}^{2}$, $x_{k}(h)=L\left(h^{k} \mathbf{1}_{X_{0}}\right)+\int_{T \times X_{0}} h^{k}(t, x) \nu(d t d x), k=3,4, \ldots$ and $h \in K$. Then it holds that

$$
n ! P_{n}(\bar{x}(h))=I_{n}\left(h^{\otimes n}\right),
$$

where $h^{\otimes n}\left(t_{1}, x_{1}, \ldots, t_{n}, x_{n}, \omega\right)=h\left(t_{1}, x_{1}, \omega\right) \cdots h\left(t_{n}, x_{n}, \omega\right)$. 
Proof. We will prove the theorem by induction on $n$. For $n=1$ it is immediate. Assume it holds for $1,2, \ldots, n$. Using the product formula (19) and recursive relation for generalized orthogonal polynomials (10), we have

$$
\begin{aligned}
& I_{n+1}\left(h^{\otimes(n+1)}\right)= \\
& I_{n}\left(h^{\otimes n}\right) I_{1}(h)-n I_{n-1}\left(h^{\otimes(n-1)} \pi\left(h^{2}\right)\right)-n I_{n}\left(h^{\otimes(n-1)} \otimes\left(h^{2} \mathbf{1}_{X_{0}}\right)\right) \\
& =n ! P_{n}(\bar{x}(h)) L(h)-n ! \pi\left(h^{2}\right) P_{n-1}(\bar{x}(h))-n I_{n-1}\left(h^{\otimes(n-1)}\right) I_{1}\left(h^{2} \mathbf{1}_{X_{0}}\right) \\
& +n(n-1) I_{n-2}\left(h^{\otimes(n-2)}\right) \pi\left(h^{3} \mathbf{1}_{X_{0}}\right)+n(n-1) I_{n-1}\left(h^{\otimes(n-2)} \otimes\left(h^{3} \mathbf{1}_{X_{0}}\right)\right) \\
& =n ! \sum_{k=0}^{1}(-1)^{k+1} x_{k+1}(h) P_{n-k}(\bar{x}(h))+n ! P_{n-2}(\bar{x}(h)) \pi\left(h^{3} \mathbf{1}_{X_{0}}\right) \\
& \quad+n(n-1) I_{n-1}\left(h^{\otimes(n-2)} \otimes\left(h^{3} \mathbf{1}_{X_{0}}\right)\right)=\ldots \\
& =n ! \sum_{k=0}^{n-1}(-1)^{k+1} x_{k+1}(h) P_{n-k}(\bar{x}(h))+n !(-1)^{n} P_{0}(\bar{x}(h)) \pi\left(h^{n+1} \mathbf{1}_{X_{0}}\right) \\
& \quad+n !(-1)^{n} I_{1}\left(h^{n+1}\right)=n ! \sum_{k=0}^{n}(-1)^{k+1} x_{k+1}(h) P_{n-k}(\bar{x}(h)) \\
& =(n+1) ! P_{n+1}(\bar{x}(h)),
\end{aligned}
$$

which completes the proof of the theorem.

From this theorem and Theorem 10 we deduce the following result.

Corollary 14. Any square integrable random variable $\xi \in L^{2}(\Omega, \mathcal{F}, \mathrm{P})$ can be expanded into a series of multiple stochastic integrals:

$$
\xi=\sum_{k=0}^{\infty} I_{k}\left(f_{k}\right) .
$$

Here $f_{0}=\mathrm{E}[\xi \mid \mathcal{H}]$, and $I_{0}$ is the identity mapping on the $L^{2}(\Omega, \mathcal{H}, \mathrm{P})$. Furthermore, this representation is unique provided the functions $f_{k} \in L^{2}\left(M_{\pi}^{k}\right)$ are symmetric with respect to the pairs of nonrandom variables.

Proof. The proof uses the same arguments as those used, for example, in [22, Th. 1.1.2], so we omit it.

The following technical lemma will be needed in the sequel.

Lemma 15. Let $f_{k} \in L^{2}\left((T \times X)^{k} \times \Omega, \mathcal{G}^{\otimes k} \otimes \mathcal{H}, M_{\pi}^{k}\right)$ and $g_{m} \in L^{2}\left((T \times X)^{m} \times\right.$ $\left.\Omega, \mathcal{G}^{\otimes m} \otimes \mathcal{H}, M_{\pi}^{m}\right)$ be a symmetric with respect to pairs of nonrandom variables functions and $p \leq k \wedge m$. Then there exist $\mathcal{G}^{\otimes p} \otimes \mathcal{F}$ measurable versions of the processes $I_{k-p}\left(f_{k}\left(\cdot, t_{1}, x_{1}, \ldots, t_{p}, x_{p}\right)\right)$ and $I_{m-p}\left(g_{m}\left(\cdot, t_{1}, x_{1}, \ldots, t_{p}, x_{p}\right)\right)$ which belong to $L^{2}\left((T \times X)^{p} \times \Omega, \mathcal{G}^{\otimes p} \otimes \mathcal{F}, M_{\pi}^{p}\right)$ and the following equality holds 


$$
\begin{aligned}
\mathrm{E}\left[\int_{(T \times X)^{p}} I_{k-p}\left(f_{k}\left(\cdot, t_{1}, x_{1}, \ldots, t_{p}, x_{p}\right)\right)\right. \\
\left.\quad I_{m-p}\left(g_{m}\left(\cdot, t_{1}, x_{1}, \ldots, t_{p}, x_{p}\right)\right) \pi\left(d t_{1} d x_{1}\right) \cdots \pi\left(d t_{p} d x_{p}\right)\right] \\
=\left\{\begin{array}{cc}
0, & \text { if } m \neq k, \\
(m-p) ! \mathrm{E}\left[\int_{(T \times X)^{m}}\left(g_{m} f_{m}\right)\left(t_{1}, x_{1}, \ldots, t_{m}, x_{m}\right)\right. & \\
\left.\pi\left(d t_{1} d x_{1}\right) \cdots \pi\left(d t_{m} d x_{m}\right)\right], & \text { if } m=k .
\end{array}\right.
\end{aligned}
$$

Proof. It is easy to verify that statement of the lemma is valid for elementary functions from $\mathcal{E}_{k}$ and $\mathcal{E}_{m}$. The general case will follow by the limit argument.

Now let $T=\mathbb{R}_{+}, X_{0}=\mathbb{R}_{0}, \Delta=0$ and $L(d t d x)$ be as in Example 3. Denote by $\Sigma_{n}$ the 'increasing simplex' of $\left(\mathbb{R}_{+} \times \mathbb{R}\right)^{n}$ :

$$
\Sigma_{n}=\left\{\left(t_{1}, x_{1}, \ldots, t_{n}, x_{n}\right) \in\left(\mathbb{R}_{+} \times \mathbb{R}\right)^{n}: 0<t_{1}<\cdots<t_{n}\right\},
$$

and we extend a function $f_{n}$ defined on $\Sigma_{n} \times \Omega$ by making $f_{n}$ symmetric with respect to pairs of nonrandom variables. If the function $f_{n}$ square integrable with respect to measure $M_{\pi}^{n}$ then we have

$$
I_{n}\left(f_{n}\right)=n ! \int_{\Sigma_{n}} f_{n}\left(t_{1}, x_{1}, \ldots, t_{n}, x_{n}\right) L\left(d t_{1} d x_{1}\right) \cdots L\left(d t_{n} d x_{n}\right) .
$$

Indeed, this equality is clear if $f_{n}$ is an elementary function of the form (18), and in the the general case equality will follow by the density argument, taking in account that iterated integral verifies the same properties as the multiple integral. In particular Lemma 15 holds for iterated integral. Note that the domain $\Sigma_{n}$ and its symmetrization do not cover $\left(\mathbb{R}_{+} \times \mathbb{R}\right)^{n}$ : we are ignoring the 'diagonal set'. Since in the beginning of this section was proved that the 'diagonal set' has $M_{\pi}^{n}$ measure zero and we consider the functions as an elements of $L^{2}$ which are the the equivalence classes, then we will always choose the representative that vanishes on the 'diagonal set'.

\section{The derivative operator}

In this section we introduce the operator $D$. Then we will show that it is equal to the Malliavin derivatives in the Gaussian case (see, e.g., [22]) and to the difference operator defined in $[24,26]$ in the Poisson case. We will also proof that the derivatives operators defined via the chaos decomposition in $[2,3,18,19,25,28,31]$ for certain Lévy processes coincide with the operator $D$.

We denote by $C_{b}^{\infty}\left(\mathbb{R}^{n}\right)$ the set of all infinitely continuously differentiable functions $f: \mathbb{R}^{n} \rightarrow \mathbb{R}$ such that $f$ and all of its partial derivatives are bounded.

Let $\mathcal{S}$ denote the class of smooth random variables such that a random variable $\xi \in \mathcal{S}$ has the form 


$$
\xi=f\left(L\left(h_{1}\right), \ldots, L\left(h_{n}\right)\right),
$$

where $f$ belongs to $C_{b}^{\infty}\left(\mathbb{R}^{n}\right), h_{1}, \ldots, h_{n}$ are in $K$, and $n \geq 1$.

Lemma 16. 1. The set $\mathcal{S}$ is dense in $L^{p}(\Omega, \mathcal{F}, \mathrm{P})$, for any $p \geq 1$.

2. The set $\{\xi h: \xi \in \mathcal{S}, h \in K\}$ is dense in $L^{2}\left(T \times X \times \Omega, \mathcal{G} \otimes \mathcal{F}, M_{\pi}\right)$.

3. The set $\left\{u e^{L(v)}: u, v \in K\right\}$ is a total set of $L^{2}\left(T \times X \times \Omega, \mathcal{G} \otimes \mathcal{F}, M_{\pi}\right)$.

Proof. 1. Let $\left\{h_{k}\right\}_{k=1}^{\infty} \subset K$ be a dense subset of $H$. Define $\mathcal{F}_{n}=\sigma\left(L\left(h_{1}\right), \ldots\right.$, $L\left(h_{n}\right)$ ). Then $\mathcal{F}_{n} \subset \mathcal{F}_{n+1}$ and $\mathcal{F}$ is the smallest $\sigma$-algebra containing all the $\mathcal{F}_{n}$ 's. Choose a $g \in L^{p}(\Omega)$. Then

$$
g=\mathrm{E}(g \mid \mathcal{F})=\lim _{n \rightarrow \infty} \mathrm{E}\left(g \mid \mathcal{F}_{n}\right) .
$$

By the Doob-Dynkin Lemma we have that for each $n$, there exist a Borel measurable function $g_{n}: \mathbb{R}^{n} \rightarrow \mathbb{R}$ such that

$$
\mathrm{E}\left(g \mid \mathcal{F}_{n}\right)=g_{n}\left(L\left(h_{1}\right), \ldots, L\left(h_{n}\right)\right) .
$$

Each such $g_{n}$ can be approximated by functions $f_{m}^{(n)}$ where $f_{m}^{(n)} \in C_{b}^{\infty}\left(\mathbb{R}^{n}\right)$ such that $\left\|f_{m}^{(n)}\left(L\left(h_{1}\right), \ldots, L\left(h_{n}\right)\right)-g_{n}\left(L\left(h_{1}\right), \ldots, L\left(h_{n}\right)\right)\right\|_{L^{p}(\Omega)}$ converges to zero as $m \rightarrow \infty$. Since $f_{m}^{(n)}\left(L\left(h_{1}\right), \ldots, L\left(h_{n}\right)\right) \in \mathcal{S}$ we have the first statement of the lemma.

2. It is enough to show that indicator function $\mathbf{1}_{A \times B}$, where $A \in \mathcal{G}, B \in \mathcal{F}$ and $M_{\pi}(A \times B)<\infty$ can be approximated by the processes of the form $\xi h$, where $\xi \in \mathcal{S}$ and $h \in K$. It follows from the previous part of the lemma that there exists the sequence $\xi_{n}$ in $\mathcal{S}$ such that $\xi_{n} \rightarrow \mathbf{1}_{B}$ as $n \rightarrow \infty$ in $L^{2}(\Omega)$. Set $C_{m}=\{\pi(A) \leq m\}$. Then $C_{m} \in \mathcal{H}$ and $\bigcup_{m \geq 1} C_{m}=\{\pi(A)<\infty\} \supset B$ a.s. The processes $\mathbf{1}_{A \times C_{m}} \xi_{n}$ have required form and letting $m \rightarrow \infty$ and then $n \rightarrow \infty$ we obtain the desired result.

3. Lemma 9 implies that finite linear combinations of the random variables $e^{L(v)}, v \in K$ are dense in $L^{2}(\Omega)$. The same arguments as in previous part of the lemma yield the density of the set of the linear combinations of the processes $u e^{L(v)}, u, v \in K$, which completes the proof of the lemma.

Definition 17. The stochastic derivative of a smooth random variable $\xi$ of the form (29) is the stochastic process $D \xi=\left\{D_{t, x} \xi,(t, x) \in T \times X\right\}$ indexed by the parameter space $T \times X$ given by

$$
\begin{gathered}
D_{t, x} \xi=\sum_{k=1}^{n} \frac{\partial f}{\partial y_{k}}\left(L\left(h_{1}\right), \ldots, L\left(h_{n}\right)\right) h_{k}(t, x) \mathbf{1}_{\Delta}(x) \\
+\left(f\left(L\left(h_{1}\right)+h_{1}(t, x), \ldots, L\left(h_{n}\right)+h_{n}(t, x)\right)-f\left(L\left(h_{1}\right), \ldots, L\left(h_{n}\right)\right)\right) \mathbf{1}_{X_{0}}(x) .
\end{gathered}
$$

Remark 18. 1. If the measure $\nu$ is zero and the measure $\mu$ is deterministic then $D \xi$ coincides with the Malliavin derivative (see, for example, [22, Def. 1.2.1, p. 24]). 
2. If the measure $\mu$ is zero and the measure $\nu$ is deterministic then $D$ coincides with the difference operator defined in [26].

3. If $T=\mathbb{R}_{+}$, the measure $\mu$ is the Lebesgue measure and $X$ is a metric space, and the measure $\nu$ is the product of the Lebesgue measure times the measure $\beta$ satisfying $\int_{M}\left(|x|^{2} \wedge 1\right) \beta(d x)$, then $D$ is the operator $\nabla^{-}$ from [28].

4. If measures $\mu$ and $\nu$ are both deterministic then $D$ coincides with operator defined in [33], see also [31].

Lemma 19. Suppose that $\xi$ is smooth functional of the form (29) and $h \in H$.

Then

$$
\mathrm{E}\left[\int_{T \times X} D_{t, x} \xi h(t, x) \pi(d t d x) \mid \mathcal{H}\right]=\mathrm{E}[\xi L(h) \mid \mathcal{H}] .
$$

Proof. The proof will be done in three steps.

Step 1. Suppose first that

$$
\xi=e^{i z_{1} L\left(h_{1}\right)} \cdots e^{i z_{n} L\left(h_{n}\right)} .
$$

Then $\operatorname{Re} \xi \in \mathcal{S}$ and $\operatorname{Im} \xi \in \mathcal{S}$ and

$$
\begin{gathered}
\mathrm{E}[\xi L(h) \mid \mathcal{H}]=\left.\frac{1}{i} \frac{d}{d z}\left(\mathrm{E}\left[\exp \left(i \sum_{k=1}^{n} z_{k} L\left(h_{k}\right)+i z L(h)\right) \mid \mathcal{H}\right]\right)\right|_{z=0} \\
=\frac{1}{i} \frac{d}{d z} \exp \left(-\frac{1}{2} \int_{T}\left(\sum_{k=1}^{n} z_{k} h_{k}(t, \Delta)+z h(t, \Delta)\right)^{2} \mu(d t)\right. \\
+\int_{T \times X_{0}}\left(\exp \left(i \sum_{k=1}^{n} z_{k} h_{k}(t, x)+i z h(t, x)\right)-1\right. \\
\left.\left.-i\left(\sum_{k=1}^{n} z_{k} h_{k}(t, x)+z h(t, x)\right)\right) \nu(d t d x)\right)\left.\right|_{z=0} \\
=\left(\int_{T \times X_{0}} h(t, x)\left(\exp \left(i \sum_{k=1}^{n} z_{k} h_{k}(t, x)\right)-1\right) \nu(d t d x)\right. \\
\left.+i \int_{T} h(t, \Delta) \sum_{k=1}^{n} z_{k} h_{k}(t, \Delta) \mu(d t)\right) \exp \left(-\frac{1}{2} \int_{T}\left(\sum_{k=1}^{n} z_{k} h_{k}(t, \Delta)\right)^{2} \mu(d t)\right. \\
\left.\left.\left.+\int_{T \times X_{0}}\left(\exp \left(i \sum_{k=1}^{n} z_{k} h_{k}(t, x)\right)-1-i \sum_{k=1}^{n} z_{k} h_{k}(t, x)\right) \nu(d t d x)\right)^{n} z_{k=1}^{n} z_{k} h_{k}(t, x)\right)-1\right) \nu(d t d x) \\
=\mathrm{E}[\xi \mid \mathcal{H}]\left(\int _ { T \times X _ { 0 } } h ( t , x ) \left(\exp \left(i \sum_{k}\right)\right.\right.
\end{gathered}
$$




$$
\left.+i \int_{T} h(t, \Delta) \sum_{k=1}^{n} z_{k} h_{k}(t, \Delta) \mu(d t)\right) .
$$

On the other hand

$$
\begin{aligned}
& \mathrm{E}\left[\int_{T \times X} D_{t, x} \xi h(t, x) \pi(d t d x) \mid \mathcal{H}\right] \\
& =\mathrm{E}\left[\int_{T \times X_{0}}\left(\exp \left(i \sum_{k=1}^{n} z_{k}\left(L\left(h_{k}\right)+h_{k}(t, x)\right)\right)-\exp \left(i \sum_{k=1}^{n} z_{k} L\left(h_{k}\right)\right)\right)\right. \\
& h(t, x) \nu(d t d x) \mid \mathcal{H}]+\mathrm{E}\left[\int_{T} i \sum_{j=1}^{n} z_{j} \exp \left(i \sum_{k=1}^{n} z_{k} L\left(h_{k}\right)\right) h_{j}(t, \Delta) \mu(d t) \mid \mathcal{H}\right] \\
& =\mathrm{E}[\xi \mid \mathcal{H}]\left(\int_{T \times X_{0}} h(t, x)\left(\exp \left(i \sum_{k=1}^{n} z_{k} h_{k}(t, x)\right)-1\right) \nu(d t d x)\right. \\
& \left.+i \int_{T} h(t, \Delta) \sum_{k=1}^{n} z_{k} h_{k}(t, \Delta) \mu(d t)\right) .
\end{aligned}
$$

Hence we have (31). By linearity we deduce that (31) also holds for smooth variables of the form (29), where the function $f$ is a trigonometric polynomial.

Step 2. Assume that $\xi$ of the form (29) such that $f \in C_{b}^{\infty}\left(\mathbb{R}^{n}\right)$ is periodic on every variable function. Then there is a sequence of trigonometric polynomials $g_{m}$ such that $g_{m} \rightarrow f$ and $\partial g_{m} / \partial x_{k} \rightarrow \partial f / \partial x_{k}$ for every $k=1, \ldots n$ uniformly on $\mathbb{R}^{n}$ as $m \rightarrow \infty$. Denote $\eta_{m}=g_{m}\left(L\left(h_{1}\right), \ldots, L\left(h_{n}\right)\right)$. Then $\eta_{m} \in \mathcal{S}$, and by Step 1 we get

$$
\mathrm{E}\left[\eta_{m} L(h) \mid \mathcal{H}\right]=\mathrm{E}\left[\int_{T \times X} D_{t, x} \eta_{m} h(t, x) \pi(d t d x) \mid \mathcal{H}\right] .
$$

Since $\eta_{m} \rightarrow \xi$ in $L^{2}(\Omega)$ and $D \eta_{m} \rightarrow D \xi$ in $L^{2}\left(T \times X \times \Omega, \mathcal{G} \otimes \mathcal{F}, M_{\pi}\right)$ then letting $m \rightarrow \infty$ in (32) we obtain (31).

Step 3. Assume that $\xi$ of the form (29). Consider the sequence $\left\{\chi_{m}, m=\right.$ $1,2, \ldots\}$ of functions, such that $\chi_{m} \in C^{\infty}\left(\mathbb{R}^{n}\right), 0 \leq \chi_{m} \leq 1, \chi_{m}(x)=1$ if $|x| \leq m, \chi(x)=0$, if $|x|>m+1$ and $\left|\nabla \chi_{m}\right| \leq 2$. Define $g_{m}$ as a periodic extension on all variables of the function $f \chi_{m}$. Then $\zeta_{m}=g_{m}\left(L\left(h_{1}\right), \ldots, L\left(h_{n}\right)\right)$ is smooth variable such that $\left|\zeta_{m}\right| \leq\|f\|_{L^{\infty}}$ and $\left|D \zeta_{m}\right| \leq\|\nabla f\|_{L^{\infty}} \sum_{i=1}^{n}\left|h_{i}\right|$. Hence by the dominated convergence theorem $\zeta_{m} \rightarrow \xi$ in $L^{2}(\Omega)$ and $D \zeta_{m} \rightarrow$ $D \xi$ in $L^{2}\left(T \times X \times \Omega, \mathcal{G} \otimes \mathcal{F}, M_{\pi}\right)$ as $m \rightarrow \infty$. Since by Step 2 formula (31) is true for $\zeta_{m}$, then letting $m \rightarrow \infty$ completes the proof of the lemma.

Applying this lemma to the product of two smooth functionals we obtain the "integration by parts" formula.

Lemma 20. Suppose $\xi$ and $\eta$ are the smooth functionals and $h \in H$, then 


$$
\begin{aligned}
& \mathrm{E}[\xi \eta L(h) \mid \mathcal{H}]= \\
& \quad \mathrm{E}\left[\int_{T \times X}\left(\xi D_{t, x} \eta+\eta D_{t, x} \xi+\mathbf{1}_{X_{0}} D_{t, x} \xi D_{t, x} \eta\right) h(t, x) \pi(d t d x) \mid \mathcal{H}\right] .
\end{aligned}
$$

As a consequence of the above lemma it can be shown in the same way as in [33] that the expression of the derivative $D \xi$ given in (30) does not depend on the particular representation of $\xi$ in (29).

For $p \geq 1$ define a norm for $\mathcal{G} \otimes \mathcal{F}$ measurable function by the following expression

$$
\|u\|_{2, p}=\left(\mathrm{E}\left[\left(\int_{T \times X} u(t, x)^{2} \pi(d t d x)\right)^{p / 2}\right]\right)^{1 / p} .
$$

Let $L^{2, p}\left(M_{\pi}\right)=L^{2, p}\left(T \times X \times \Omega, \mathcal{G} \otimes \mathcal{F}, M_{\pi}\right)$ be the set of all (equivalent classes of) functions $u(t, x, \omega)$ on $T \times X \times \Omega$ such that $\|u\|_{2, p}<\infty$.

Lemma 21. The operator $D$ is closable as an operator from $L^{p}(\Omega, \mathcal{F}, \mathrm{P})$ to $L^{2, p}\left(M_{\pi}\right)$, for any $p \geq 1$.

Proof. Let $\left\{\xi_{n}, n \geq 1\right\}$ be a sequence of smooth random variables such that $\mathrm{E}\left|\xi_{n}\right|^{p} \rightarrow 0$ and $D \xi_{n}$ converges to $\zeta$ in $L^{2, p}\left(M_{\pi}\right)$. Then from Lemma 20 it follows that for any $h \in K$ and $\eta \in \mathcal{S}$ we have

$$
\mathrm{E}\left(\xi_{n} \eta L(h)\right)=\left\langle D \eta ; \xi_{n} h\right\rangle_{L^{2}\left(M_{\pi}\right)}+\left\langle D \xi_{n} ; \eta h\right\rangle_{L^{2}\left(M_{\pi}\right)}+\left\langle D \xi_{n} ; \mathbf{1}_{X_{0}} h D \eta\right\rangle_{L^{2}\left(M_{\pi}\right)} .
$$

Taking the limit as $n \rightarrow \infty$, since $\eta, D \eta$ are bounded, and $h \in K$ we obtain

$$
\langle\zeta ; \eta h\rangle_{L^{2}\left(M_{\pi}\right)}+\left\langle\zeta ; \mathbf{1}_{X_{0}} h D \eta\right\rangle_{L^{2}\left(M_{\pi}\right)}=0 .
$$

If $h(t, x)=0$ for $x \neq \Delta$, then (34) implies, that

$$
\langle\zeta ; \eta h\rangle_{L^{2}\left(M_{\pi}\right)}=0 .
$$

Thus from Lemma 16 we deduce $\zeta_{t, \Delta}=0$ for $M_{\pi}$-almost all $(t, \Delta, \omega) \in$ $T \times\{\Delta\} \times \Omega$. Substituting this expression into (34) we have for any $h \in H$

$$
\langle\zeta ; h D \eta\rangle_{L^{2}\left(M_{\pi}\right)}=0 .
$$

Let $\phi_{n} \in C_{b}^{\infty}(\mathbb{R})$ such that $0 \leq \phi_{n}(x) \leq e^{x}$ and $\phi_{n}(x) \rightarrow e^{x}$ for all $x \in \mathbb{R}$. Putting in (35) $\eta=\phi_{n}(L(g))$ and $h(t, x)=u(t, x) e^{-g(t, x)}$, where $u, g \in K$ and then letting $n \rightarrow \infty$ we get

$$
\left\langle\zeta ; u e^{L(g)}\right\rangle_{L^{2}\left(M_{\pi}\right)}=0 .
$$

It follows from Lemma 16 that $\zeta_{t, x}=0$ for $M_{\pi}$-a.a. $(t, x, \omega) \in T \times X \times \Omega$ completing the proof of the lemma. 
We will denote the closure of $D$ again $D$ and its domain in $L^{p}(\Omega)$ by $\mathbb{D}^{1, p}$. Now we will state the chain rule.

Proposition 22. Suppose $p \geq 1$ is fixed and $\xi=\left(\xi^{1}, \ldots, \xi^{m}\right)$ is a random vector whose components belong to the space $\mathbb{D}^{1, p}$. Let $\phi \in C^{1}\left(\mathbb{R}^{m}\right)$ be a function with bounded partial derivatives. Then $\phi(\xi) \in \mathbb{D}^{1, p}$ and

$$
D_{t, x} \phi(\xi)= \begin{cases}\sum_{k=1}^{m} \frac{\partial \phi}{\partial x_{k}}(\xi) D_{t, \Delta} \xi^{k}, & \text { if } x=\Delta, \\ \phi\left(\xi^{1}+D_{t, x} \xi^{1}, \ldots, \xi^{m}+D_{t, x} \xi^{m}\right)-\phi\left(\xi^{1}, \ldots, \xi^{m}\right), & \text { if } x \neq \Delta .\end{cases}
$$

Proof. The proof can be easily obtain by approximation $\xi$ by smooth random variables and the function $\phi$ by smooth functions with compact support.

Applying the above proposition we obtain, that $L(h) \in \mathbb{D}^{1,2}$ for all $h \in H$ and $D_{t, x} L(h)=h(t, x)$.

Lemma 23. It holds that $P_{n}(\bar{x}(h)) \in \mathbb{D}^{1, p}$ for all $p \geq 1, h \in K, n=1,2, \ldots$ and

$$
D_{t, x} P_{n}(\bar{x}(h))=P_{n-1}(\bar{x}(h)) h(t, x) .
$$

Proof. As in the proof of Proposition 22 one can obtain that $P_{n}(\bar{x}(h)) \in \mathbb{D}^{1, p}$ for all $p \geq 1, h \in K, n=1,2, \ldots$ and (36) holds. Then the definition of $\bar{x}(h)$ and equality (11) imply

$$
D_{t, \Delta} P_{n}(\bar{x}(h))=\frac{\partial P_{n}}{\partial x_{1}}(\bar{x}(h)) h(t, \Delta)=P_{n-1}(\bar{x}(h)) h(t, \Delta) .
$$

It follows from the relationships (36) and (13) that for $x \neq \Delta$ we have

$$
D_{t, x} P_{n}(\bar{x}(h))=P_{n}(\bar{x}(h)+\bar{u}(h(t, x)))-P_{n}(\bar{x}(h))=h(t, x) P_{n-1}(\bar{x}(h)),
$$

where $\bar{u}(y)=\left(y, y^{2}, \ldots, y^{k}, \ldots\right)$. The proof is complete.

The product rule can be proved in the same manner.

Proposition 24. Let $\xi \in \mathbb{D}^{1, p}, p \geq 1$ and $\eta$ is a smooth variable from $\mathcal{S}$. Then $\xi \eta \in \mathbb{D}^{1, p}$ and

$$
D(\xi \eta)=\xi D \eta+\eta D \xi+D \xi D \eta \mathbf{1}_{X_{0}} .
$$

Proof. The equation (38) holds if $\xi$ and $\eta$ are smooth variables. Then, the general case follows by a limit argument, using the fact that $D$ is closed.

The following proposition is more or less evident.

Proposition 25. Let $\xi$ be $\mathcal{H}$-measurable random variable such that $\xi \in$ $L^{p}(\Omega, \mathcal{H}, \mathrm{P})$ for some $p \geq 1$. Then $\xi \in \mathbb{D}^{1, p}$ and $D \xi=0 M_{\pi}$-a.e. 
Proof. By the density arguments we can assume that $\xi=\mathbf{1}_{U}$, where $U \in \mathcal{H}$. Then for any $h \in K$ as in the proof of Lemma 7 we have $\xi \mathbf{1}_{\{L(h) \neq 0\}}=$ $L(\xi h)^{2} / L(h)^{2} \mathbf{1}_{\{L(h) \neq 0\}}$. Since $h \in K$ it easy to show that $\eta_{\varepsilon}(h)=L(\xi h)^{2} /$ $\left(L(h)^{2}+\varepsilon\right)=\xi L(h)^{2} /\left(L(h)^{2}+\varepsilon\right) \rightarrow \xi \mathbf{1}_{\{L(h) \neq 0\}}$ as $\varepsilon \rightarrow 0$ in $L^{p}(\Omega)$. If we will show that $\eta_{\varepsilon}(h) \in \mathbb{D}^{1, p}$ and $D \eta_{\varepsilon}(h) \rightarrow 0$ in $L^{2, p}\left(M_{\pi}\right)$ for any $h \in K$ then $\xi \mathbf{1}_{\{L(h) \neq 0\}}$ will be in $\mathbb{D}^{1, p}$ and $D\left(\xi \mathbf{1}_{\{L(h) \neq 0\}}\right)=0$ for any $h \in K$ implying as in the proof of Lemma 7 that $\xi \in \mathbb{D}^{1, p}$ and $D \xi=0$.

Let us show that $\eta_{\varepsilon}(h) \in \mathbb{D}^{1, p}$ and $D \eta_{\varepsilon}(h) \rightarrow 0$. Set $f(x, y)=x^{2} /\left(y^{2}+\varepsilon\right)$. Then $f(x, y) e^{-\left(x^{2}+y^{2}\right) / n}=f_{n}(x, y) \in C_{b}^{\infty}\left(\mathbb{R}^{2}\right)$ and by dominated convergence theorem we have $f_{n}(L(\xi h), L(h)) \rightarrow f(L(\xi h), L(h))=\eta_{\varepsilon}(h)$ as $n \rightarrow \infty$ in $L^{p}(\Omega)$. In the same way we obtain that the derivative $D f_{n}(L(\xi h), L(h))$ converges in $L^{2, p}\left(M_{\pi}\right)$ which implies $\eta_{\varepsilon}(h) \in \mathbb{D}^{1, p}$ and the limit $D\left(\eta_{\varepsilon}(h)\right)$ is given by

$$
\begin{aligned}
D\left(\eta_{\varepsilon}(h)\right)= & \left(\frac{2 L(\xi h) \xi h}{L(h)^{2}+\varepsilon}-\frac{2 L(h) L(\xi h)^{2} h}{\left(L(h)^{2}+\varepsilon\right)^{2}}\right) \mathbf{1}_{\Delta}+ \\
& \left(\frac{(L(\xi h)+\xi h)^{2}}{(L(h)+h)^{2}+\varepsilon}-\frac{L(\xi h)^{2}}{L(h)^{2}+\varepsilon}\right) \mathbf{1}_{X_{0}} \\
= & \left(\frac{2 \xi^{2} L(h) h}{\left(L(h)^{2}+\varepsilon\right)^{2}} \mathbf{1}_{\Delta}+\xi^{2} \frac{(L(h)+h)^{2}-L(h)^{2}}{\left((L(h)+h)^{2}+\varepsilon\right)\left(L(h)^{2}+\varepsilon\right)} \mathbf{1}_{X_{0}}\right) \varepsilon .
\end{aligned}
$$

Letting $\varepsilon \rightarrow 0$ in the equality above we obtain the desired result.

The following lemma shows the action of the operator $D$ via the chaos decomposition.

Proposition 26. Let $\xi \in L^{2}(\Omega)$ with a development

$$
\xi=\sum_{k=0}^{\infty} I_{k}\left(f_{k}\right)
$$

where $f_{k} \in L^{2}\left(M_{\pi}^{k}\right)$ are symmetric with respect to pairs of nonrandom variables. Then $\xi \in \mathbb{D}^{1,2}$ if and only if

$$
\sum_{k=1}^{\infty} k k !\left\|f_{k}\right\|_{L^{2}\left(M_{\pi}^{k}\right)}^{2}<\infty
$$

and in this case we have

$$
D_{t, x} \xi=\sum_{k=1}^{\infty} k I_{k-1}\left(f_{k}(\cdot, t, x)\right)
$$

Moreover

$$
\begin{aligned}
\mathrm{E}\left[\int_{T \times X}\left(D_{t, x} \xi\right)^{2} \pi(d t d x) \mid \mathcal{H}\right]= \\
\quad \sum_{k=1}^{\infty} k k ! \int_{(T \times X)^{k}} f_{k}\left(t_{1}, x_{1}, \ldots, t_{k}, x_{k}\right)^{2} \pi\left(d t_{1} d x_{1}\right) \cdots \pi\left(d t_{k} d x_{k}\right) .
\end{aligned}
$$


Proof. The proof will be done in three steps.

Step 1. Suppose first that $k \geq 1$ and

$$
\xi=P_{k}(\bar{x}(h))=\frac{1}{k !} I_{k}\left(h^{\otimes k}\right)=I_{k}\left(f_{k}\right),
$$

with $h \in K$. Then by Lemma $23 \xi \in \mathbb{D}^{1,2}$ and by equality (37) we get

$$
D_{t, x} P_{k}(\bar{x}(h))=P_{k-1}(\bar{x}(h)) h(t, x) .
$$

Hence

$$
D_{t, x} \xi=k I_{k-1}\left(f_{k}(\cdot, t, x)\right) .
$$

¿From Proposition 25 and formula (38) we deduce that equality (43) holds for any linear combination of random variables of the form $\eta P_{k}(\bar{x}(h))$, where $\eta$ is $\mathcal{H}$-measurable bounded random variable. Since formula (43) implies that $\|D \xi\|_{L^{2}\left(M_{\pi}\right)}^{2}=k \mathrm{E} \xi^{2}$ then it follows that $\mathcal{P}_{k}, k \geq 1$ is included in $\mathbb{D}^{1,2}$.

If $k=0$ then Proposition 25 implies that $\mathcal{P}_{0}=L^{2}(\Omega, \mathcal{H}, \mathrm{P}) \subset \mathbb{D}^{1,2}$.

Step 2. Let $\xi \in L^{2}(\Omega)$ has an expansion (39). Suppose that (40) holds. Define

$$
\xi_{n}=\sum_{k=0}^{n} I_{k}\left(f_{k}\right) .
$$

Then the sequence $\xi_{n}$ converges to $\xi$ in $L^{2}(\Omega)$, and by Step 1 we have $\xi_{n} \in \mathbb{D}^{1,2}$ and $D_{t, x} \xi=\sum_{k=1}^{n} k I_{k-1}\left(f_{k}(\cdot, t, x)\right)$. It follows from Lemma 15 and equality (40) that $D_{t, x} \xi_{n}$ converges in $L^{2}\left(M_{\pi}\right)$ to the right-hand side of (41). Therefore $\xi \in \mathbb{D}^{1,2}$ and (41) holds.

Step 3. Suppose $\xi \in \mathbb{D}^{1,2}$. Note that formula (33) holds for $\xi \in \mathbb{D}^{1,2}$ and $\eta \in \mathbb{D}^{1, p}$ for some $p>2$ if $h \in K$. Since by Proposition $23 \eta=P_{m}(\bar{x}(g)) \in \mathbb{D}^{1, p}$ for all $p \geq 1$ and $g \in K$, then we have

$$
\begin{aligned}
& \lim _{n \rightarrow \infty}\left(\left\langle D \xi_{n} ; \eta h\right\rangle_{L^{2}\left(M_{\pi}\right)}+\left\langle D \xi_{n} ; D \eta h \mathbf{1}_{X_{0}}\right\rangle_{L^{2}\left(M_{\pi}\right)}\right) \\
& =\lim _{n \rightarrow \infty}\left(\mathrm{E}\left(\xi_{n} \eta L(h)\right)-\left\langle D \eta ; \xi_{n} h\right\rangle_{L^{2}\left(M_{\pi}\right)}\right) \\
& =\mathrm{E}(\xi \eta L(h))-\langle D \eta ; \xi h\rangle_{L^{2}\left(M_{\pi}\right)}=\langle D \xi ; \eta h\rangle_{L^{2}\left(M_{\pi}\right)}+\left\langle D \xi ; D \eta h \mathbf{1}_{X_{0}}\right\rangle_{L^{2}\left(M_{\pi}\right)} .
\end{aligned}
$$

It follows from equation (23) that

$$
\eta+\mathbf{1}_{X_{0}} D \eta=P_{m}(\bar{x}(g))+\mathbf{1}_{X_{0}} g P_{m-1}(\bar{x}(g)) .
$$

Then for all $m=1,2, \ldots$ we obtain

$$
\begin{gathered}
\lim _{n \rightarrow \infty}\left(\left\langle D \xi_{n} ; P_{m}(\bar{x}(g)) h\right\rangle_{L^{2}\left(M_{\pi}\right)}+\left\langle D \xi_{n} ; P_{m-1}(\bar{x}(g)) g h \mathbf{1}_{X_{0}}\right\rangle_{L^{2}\left(M_{\pi}\right)}\right) \\
\quad=\left\langle D \xi ; P_{m}(\bar{x}(g)) h\right\rangle_{L^{2}\left(M_{\pi}\right)}+\left\langle D \xi ; P_{m-1}(\bar{x}(g)) g h \mathbf{1}_{X_{0}}\right\rangle_{L^{2}\left(M_{\pi}\right)} .
\end{gathered}
$$

Since $P_{0}=1$ and $\lim _{n \rightarrow \infty}\left\langle D \xi_{n} ; P_{0}(\bar{x}(g)) h\right\rangle_{L^{2}\left(M_{\pi}\right)}=\left\langle D \xi ; P_{0}(\bar{x}(g)) h\right\rangle_{L^{2}\left(M_{\pi}\right)}$ for all $h \in L^{2}\left(M_{\pi}\right)$, then we deduce by induction that 


$$
\lim _{n \rightarrow \infty}\left\langle D \xi_{n} ; P_{m}(\bar{x}(g)) h\right\rangle_{L^{2}\left(M_{\pi}\right)}=\left\langle D \xi ; P_{m}(\bar{x}(g)) h\right\rangle_{L^{2}\left(M_{\pi}\right)} .
$$

From Lemma 15 we deduce that for $n>m$ the expression

$$
\left\langle D \xi_{n} ; P_{m}(\bar{x}(g)) h\right\rangle_{L^{2}\left(M_{\pi}\right)}
$$

is equal to

$$
\mathrm{E}\left(\int_{T \times X}(m+1) I_{m}\left(f_{m+1}(\cdot, t, x)\right) h(t, x) \pi(d t d x) P_{m}(\bar{x}(g))\right) .
$$

Hence the projection of $\int_{T \times X} D_{t, x} \xi h(t, x) \pi(d t d x)$ on the $m$-th chaos is equal to

$$
\int_{T \times X}(m+1) I_{m}\left(f_{m+1}(\cdot, t, x)\right) h(t, x) \pi(d t d x) .
$$

Thus for any $\zeta \in L^{2}(\Omega, \mathcal{F}, \mathrm{P})$ we have

$$
\begin{gathered}
\langle D \xi ; h \zeta\rangle_{L^{2}\left(M_{\pi}\right)}=\mathrm{E}\left(\int_{T \times X} D_{t, x} \xi h(t, x) \pi(d t d x) \zeta\right) \\
=\mathrm{E}\left(\sum_{m=0}^{\infty} \int_{T \times X}(m+1) I_{m}\left(f_{m+1}(\cdot, t, x)\right) h(t, x) \pi(d t d x) \zeta\right) \\
=\left\langle\sum_{m=0}^{\infty}(m+1) I_{m}\left(f_{m+1}(\cdot, t, x)\right) ; h \zeta\right\rangle_{L^{2}\left(M_{\pi}\right)} .
\end{gathered}
$$

Since the set $\left\{h \zeta: h \in K, \zeta \in L^{2}(\Omega, \mathcal{F}, \mathrm{P})\right\}$ is dense in $L^{2}(T \times X \times \Omega, \mathcal{G} \otimes$ $\left.\mathcal{F}, M_{\pi}\right)$ then

$$
D_{t, x} \xi=\sum_{m=0}^{\infty}(m+1) I_{m}\left(f_{m+1}(\cdot, t, x)\right),
$$

which completes the proof of the proposition.

Remark 2\%. This proposition implies that the operator $D$ is an annihilation operator on the Fock space on Hilbert space $H$.

The equations (41) can be considered as a definition of the operator $D$. This approach was developed for pure jump Lévy process, the particular case of Poisson processes, the case of general Lévy process with no drift and the case of certain class of martingales in [2, 3, 18, 19, 25, 28].

Let $A \in \mathcal{G} \otimes \mathcal{H}$. We will denote by $\mathcal{F}_{A}^{0}$ the $\sigma$-algebra generated by the random variables $\left\{L(B), B \subset A, B \in \mathcal{G}_{0}\right\}$. Set $\mathcal{F}_{A}=\mathcal{F}_{A}^{0} \vee \mathcal{H}$. The following results are modification of Proposition 1.2.5 from [22, p. 32] and it shows how to compute the derivative of a conditional expectation with respect to a $\sigma$-algebra generated by stochastic process. 
Lemma 28. Suppose that $\xi \in L^{2}(\Omega, \mathcal{F}, \mathrm{P})$ with the expansion (39). Let $A \in$ $\mathcal{G} \otimes \mathcal{H}$. Then

$$
\mathrm{E}\left[\xi \mid \mathcal{F}_{A}\right]=\sum_{k=0}^{\infty} I_{k}\left(f_{k} \mathbf{1}_{A}^{\otimes k}\right) .
$$

Proof. By the density of elementary functions in $L^{2}\left(M_{\pi}^{k}\right)$ and by linearity we can assume that $\xi=I_{k}\left(f_{k}\right)$, where $f_{k}=\eta \mathbf{1}_{A_{1}} \otimes \cdots \otimes \mathbf{1}_{A_{k}}$ with pairwise-disjoint sets $A_{1}, \ldots, A_{k} \in \mathcal{G}_{0}$ and $\eta \in L^{\infty}(\Omega, \mathcal{H}, \mathrm{P})$. Then we have

$$
\begin{gathered}
\mathrm{E}\left[\xi \mid \mathcal{F}_{A}\right]=\mathrm{E}\left[\eta L\left(A_{1}\right) \cdots L\left(A_{k}\right) \mid \mathcal{F}_{A}\right] \\
=\eta \mathrm{E}\left[\prod_{i=1}^{k}\left(L\left(A_{i} \cap A\right)+L\left(A_{i} \backslash A\right)\right) \mid \mathcal{F}_{A}\right]=\eta \mathrm{E}\left[L\left(A_{1} \cap A\right) \cdots L\left(A_{m} \cap A\right) \mid \mathcal{F}_{A}\right]= \\
=I_{k}\left(\eta \mathbf{1}_{A_{1} \cap A} \otimes \cdots \otimes \mathbf{1}_{A_{k} \cap A}\right)=I_{k}\left(f_{k} \mathbf{1}_{A}^{\otimes k}\right) .
\end{gathered}
$$

Proposition 29. Suppose that $\xi \in \mathbb{D}^{1,2}$, and $A \in \mathcal{G} \otimes \mathcal{H}$. Then $\mathrm{E}\left(\xi \mid \mathcal{F}_{A}\right) \in \mathbb{D}^{1,2}$ and we have

$$
D_{t, x}\left(\mathrm{E}\left(\xi \mid \mathcal{F}_{A}\right)\right)=\mathrm{E}\left(D_{t, x} \xi \mid \mathcal{F}_{A}\right) \mathbf{1}_{A}(t, x)
$$

$M_{\pi}$-a.e. in $T \times X \times \Omega$.

Proof. By Lemma 28 and Proposition 26 we obtain

$\mathrm{E}\left(D_{t, x} \xi \mid \mathcal{F}_{A}\right) \mathbf{1}_{A}(t, x)=\sum_{k=1}^{\infty} k I_{k-1}\left(f_{k}(\cdot, t, x) \mathbf{1}_{A}^{\otimes(k-1)}\right) \mathbf{1}_{A}(t, x)=D_{t, x}\left(\mathrm{E}\left(\xi \mid \mathcal{F}_{A}\right)\right)$.

Remark 30. In particular, if $\xi$ is $\mathcal{F}_{A^{-}}$-measurable and belongs to $\mathbb{D}^{1,2}$, then $D_{t, x} \xi=0 M_{\pi}$-a.e. in $A^{c}$.

\section{The Skorohod integral}

In this section we consider the adjoint of the operator $D$, and we will show that it coincides with the Skorohod integral [30] in the Gaussian case and with the extended stochastic integral introduced by Kabanov [13] in the pure jump Lévy case. See also $[2,3,18,28]$. So it can be considered as a generalization of the stochastic integral. We will call it Skorohod integral and will establish the expression of it in terms of the chaos expansion as well as prove some of its properties.

We recall that the derivative operator $D$ is a closed and unbounded operator defined on the dense subset $\mathbb{D}^{1,2}$ of $L^{2}(\Omega)$ with values in $L^{2}(T \times X \times$ $\left.\Omega, \mathcal{G} \otimes \mathcal{F}, M_{\pi}\right)$.

Definition 31. We denote by $\delta$ the adjoint of the operator $D$ and will call it Skorohod integral. 
The operator $\delta$ is closed unbounded operator on $L^{2}\left(T \times X \times \Omega, \mathcal{G} \otimes \mathcal{F}, M_{\pi}\right)$ with values in $L^{2}(\Omega)$ defined on Dom $\delta$, where Dom $\delta$ is the set of processes $u \in L^{2}\left(M_{\pi}\right)$ such that

$$
\left|\mathrm{E} \int_{T \times X} D_{t, x} \xi u(t, x) \pi(d t d x)\right| \leq c\|\xi\|_{L^{2}(\Omega)}
$$

for all $\xi \in \mathbb{D}^{1,2}$, where $c$ is some constant depending on $u$.

If $u \in \operatorname{Dom} \delta$, then $\delta(u)$ is the element of $L^{2}(\Omega)$ such that

$$
\mathrm{E}(\xi \delta(u))=\mathrm{E} \int_{T \times X} D_{t, x} \xi u(t, x) \pi(d t d x)
$$

for any $\xi \in \mathbb{D}^{1,2}$.

The following proposition shows the behavior of $\delta$ in terms of the chaos expansion.

Proposition 32. Let $u \in L^{2}\left(T \times X \times \Omega, \mathcal{G} \otimes \mathcal{F}, M_{\pi}\right)$ with the expansion

$$
u(t, x)=\sum_{k=0}^{\infty} I_{k}\left(f_{k}(\cdot, t, x)\right) .
$$

Then $u \in \operatorname{Dom} \delta$ if and only if the series

$$
\delta(u)=\sum_{k=0}^{\infty} I_{k+1}\left(\tilde{f}_{k}\right)
$$

converges in $L^{2}(\Omega)$.

Recall that $\tilde{f}_{k}$ is a symmetrization of $f_{k}$ in all its pairs of nonrandom variables is given by

$$
\begin{gathered}
\tilde{f}_{k}\left(t_{1}, x_{1}, \ldots, t_{k}, x_{k}, t, x, \omega\right)=\frac{1}{k+1}\left(f_{k}\left(t_{1}, x_{1}, \ldots, t_{k}, x_{k}, t, x, \omega\right)\right. \\
\left.+\sum_{i=1}^{k} f_{k}\left(t_{1}, x_{1}, \ldots, t_{i-1}, x_{i-1}, t, x, t_{i+1}, x_{i+1}, \ldots, t_{i}, x_{i}, \omega\right)\right) .
\end{gathered}
$$

Proof. The proof is the same as in the Gaussian case (see, e.g., [22, Prop. 1.3.1, p. 36]).

Remark 33. It follows from Proposition 32 that the operator $\delta$ coincides with Skorohod integral in the Gaussian case and with extended stochastic integral introduced by Kabanov for pure jump Lévy processes (see, e.g., [30, 13, 22, 2, $3,18,28])$. 
It follows from proposition above that Dom $\delta$ is the subspace of $L^{2}\left(M_{\pi}\right)$ formed by the processes that satisfy the following condition:

$$
\sum_{k=1}^{\infty}(k+1) !|| \tilde{f}_{k} \|_{L^{2}\left(M_{\pi}^{k+1}\right)}^{2}<\infty .
$$

If $u \in \operatorname{Dom} \delta$, then the sum of the series (48) is equal to $\mathrm{E} \delta(u)^{2}$.

Note that the Skorohod integral is a linear operator and has zero mean, e.g., $\mathrm{E}(\delta(u))=0$ if $u \in \operatorname{Dom} \delta$. The following statements prove some properties of $\delta$.

Proposition 34. Let $u, v \in$ Dom $\delta$ be arbitrary stochastic process. Then for all $\alpha$ and $\beta$ in $L^{\infty}(\Omega, \mathcal{H}, \mathrm{P})$ we have $\alpha u+\beta v \in \operatorname{Dom} \delta$ and

$$
\delta(\alpha u+\beta v)=\alpha \delta(u)+\beta \delta(v) .
$$

Moreover $\mathrm{E}[\delta(u) \mid \mathcal{H}]=0$.

Proof. The proof follows from the properties (i) and (iii) of the multiple integral.

Proposition 35. Suppose that $u$ is a Skorohod integrable process. Let $\xi \in \mathbb{D}^{1,2}$ such that $\mathrm{E}\left(\int_{T \times X}\left(\xi^{2}+\left(D_{t, x} \xi\right)^{2} \mathbf{1}_{X_{0}}\right) u(t, x)^{2} \pi(d t d x)\right)<\infty$. Then it holds that

$$
\delta\left(\left(\xi+\mathbf{1}_{X_{0}} D \xi\right) u\right)=\xi \delta(u)-\int_{T \times X}\left(D_{t, x} \xi\right) u(t, x) \pi(d t d x),
$$

provided that one of the two sides of the equality (49) exists.

Proof. Let $\eta \in \mathcal{S}$ be a smooth random variables. Then by the product rule (38) and by the duality relation (45), we get

$$
\begin{gathered}
\mathrm{E}\left(\int_{T \times X}\left(D_{t, x} \eta\right)\left(\xi+\mathbf{1}_{X_{0}}(x) D_{t, x} \xi\right) u(t, x) \pi(d t d x)\right) \\
=\int_{T \times X} \mathrm{E}\left(u(t, x)\left(D_{t, x}(\xi \eta)-\eta D_{t, x} \xi\right)\right) \pi(d t d x) \\
=\mathrm{E}\left(\eta\left(\xi \delta(u)-\int_{T \times X}\left(D_{t, x} \xi\right) u(t, x) \pi(d t d x)\right)\right),
\end{gathered}
$$

and the result follows.

As in the Gaussian case or in the case of processes with independent increments in order to prove some other properties of Skorohod integral we will define a class of processes contained in Dom $\delta$ (see [22], [33]).

Definition 36. Let $\mathbb{L}^{1,2}$ denote the class of processes $u \in L^{2}(T \times X \times \Omega, \mathcal{G} \otimes$ $\left.\mathcal{F}, M_{\pi}\right)$ such that $u(t, x) \in \mathbb{D}^{1,2}$ for all $(t, x) \notin R$, where $R \subset T \times X$ and $M_{\pi}(R \times \Omega)=0$, and there exists a measurable version of the multiparametrical process $D_{t, x} u(s, y)$ satisfying $\mathrm{E} \int_{T \times X} \int_{T \times X}\left(D_{t, x} u(s, y)\right)^{2} \pi(d t d x) \pi(d s d y)<$ $\infty$. 
If the process $u$ has the expansion (46), then $u \in \mathbb{L}^{1,2}$ if and only if the series

$$
\begin{aligned}
\int_{T \times X} \int_{T \times X} \mathrm{E}\left(\sum_{k=1}^{\infty} k I_{k-1}\left(f_{k}(\cdot, t, x, s, y)\right)\right)^{2} \pi(d t d x) & \pi(d s d y) \\
& =\sum_{k=1}^{\infty} k k !\left\|f_{k}\right\|_{L^{2}\left(M_{\pi}^{k+1}\right)}^{2}
\end{aligned}
$$

converges.

Since $\left\|\tilde{f}_{k}\right\|_{L^{2}\left(M_{\pi}^{k+1}\right)} \leq\left\|f_{k}\right\|_{L^{2}\left(M_{\pi}^{k+1}\right)}$ then from (48) we deduce that $\mathbb{L}^{1,2} \subset$ Dom $\delta$.

The proofs of the following propositions use the chaos expansion therefore they can be done as in the Gaussian case (see, for instance [22, pp. 38 - 40]).

Proposition 37. Suppose that $u \in \mathbb{L}^{1,2}$ and for all $(t, x) \notin R$, where $R \subset T \times X$ and $M_{\pi}(R \times \Omega)=0$ the two-parameter process $\left\{D_{t, x} u(s, y),(s, y) \in\right.$ $T \times X\}$ is Skorohod integrable, and there exists a version of the process $\left\{\delta\left(D_{t, x} u(\cdot, \cdot)\right),(t, x) \in T \times X\right\}$ which belongs to $L^{2}\left(M_{\pi}\right)$. Then $\delta(u) \in \mathbb{D}^{1,2}$ and we have

$$
D_{t, x} \delta(u)=u(t, x)+\delta\left(D_{t, x} u(\cdot, \cdot)\right) .
$$

Proposition 38. Suppose that $u \in \mathbb{L}^{1,2}$ and $v \in \mathbb{L}^{1,2}$. Then we have

$$
\begin{gathered}
\mathrm{E}[\delta(u) \delta(v) \mid \mathcal{H}]=\mathrm{E}\left[\int_{T \times X} u(t, x) v(t, x) \pi(d t d x) \mid \mathcal{H}\right] \\
+\mathrm{E}\left[\int_{T \times X} \int_{T \times X} D_{s, y} u(t, x) D_{t, x} v(s, y) \pi(d t d x) \pi(d s d y) \mid \mathcal{H}\right] .
\end{gathered}
$$

Now we will show that the operator $\delta$ is an extension of the Itô integral. Let $L_{t}, t \in[0 ; 1]$ be a processes with $\mathcal{H}$-conditionally independent increments. Assume that the canonical triplet of its characteristics $(B, \mu, \nu)$ such that $B=0$. Then as in Example 3 we have random measure $N(d t d x)$ associated to jumps of $L$ with compensator measure $\nu$, the measure $\mu$ connected with continuous part of $L$ and conditional additive process $L(h)$ on $H$. We denote by $L_{p}^{2}$ the subset of $L^{2}\left(M_{\pi}\right)$ formed by $\mathcal{F}_{t}$-predictable processes.

Te following technical will be needed.

Lemma 39. Let $A \in \mathcal{G} \otimes \mathcal{H}$ be a set with finite $M_{\pi}$ measure, and let $\xi$ be a square integrable random variable that is measurable with respect to the $\sigma$ algebra $\mathcal{F}_{A^{c}}$. Then the process $\xi \mathbf{1}_{A}$ is Skorohod integrable and

$$
\delta\left(\xi \mathbf{1}_{A}\right)=\xi L(A) .
$$

Proof. Suppose first that $\xi \in \mathbb{D}^{1,2}$ and $\mathbf{1}_{A} \in K$. By using Proposition 35 and Remark 30 we have 
$\delta\left(\xi \mathbf{1}_{A}\right)=\delta\left(\left(\xi+\mathbf{1}_{X_{0}} D \xi\right) \mathbf{1}_{A}\right)=\xi \delta\left(\mathbf{1}_{A}\right)-\int_{T \times X}\left(D_{t, x} \xi\right) \mathbf{1}_{A}(t, x) \pi(d t d x)=\xi L(A)$.

The general case follows by a limit argument, using the facts that $\mathbb{D}^{1,2}$ and $K$ are dense and $\delta$ is closed.

Proposition 40. $L_{p}^{2} \subset$ Dom $\delta$, and the restriction of the operator $\delta$ to the space $L_{p}^{2}$ coincides with the usual stochastic integral, that is

$$
\delta(u)=\int_{0}^{1} u(t, 0) d L^{c}(t)+\int_{0}^{1} \int_{\mathbb{R}_{0}} u(t, x)(N(d t d x)-\nu(d t d x)) .
$$

Proof. Suppose that $u$ is an elementary adapted processes of the form

$$
u_{t, x}=\sum_{i=1}^{n} \xi_{i} \mathbf{1}_{A_{i}} \mathbf{1}_{\left(t_{i} ; t_{i+1}\right]}(t) \mathbf{1}_{B_{i}}(x),
$$

where $0 \leq t_{1}<\cdots<t_{n+1} \leq 1, B_{i}$ is a borel set of $\mathbb{R}, A_{i} \in \mathcal{H}$ such that $\mathbf{1}_{\left(t_{i} ; t_{i+1}\right] \times B_{i} \times A_{i}}(t, x) \in K$ and $\xi_{i}$ is square integrable and $\mathcal{F}_{t_{i}}$ measurable random variable. Then from the Lemma 39 we obtain $u \in \operatorname{Dom} \delta$ and

$$
\delta(u)=\sum_{i=1}^{\infty} \xi_{i}\left(\left(L_{t_{i+1}}-L_{t_{i}}\right) \mathbf{1}_{B_{i}}(0)+\int_{t_{i}}^{t_{i+1}} \int_{B_{i}}(N(d t d x)-\nu(d t d x)) .\right.
$$

The general case follows by monotone class argument since $\delta$ is closed.

The predictable projection of a stochastic process indexed by $t \geq 0$ and $x \in$ $\mathbb{R}$ can be defined similarly as in a one parametrical case (see, i.g, $[12,17,31]$ ). Let $Y=\{Y(t, x), t \geq 0, x \in \mathbb{R}\}$ be an measurable integrable process. There exists a predictable process $Z=\{Z(t, x), t \geq 0, x \in \mathbb{R}\}$ such that for every predictable stopping time $\tau$

$$
Z(\tau, x) \mathbf{1}_{\{\tau<\infty\}}=\mathrm{E}\left[Y(\tau, x) \mathbf{1}_{\{\tau<\infty\}} \mid \mathcal{F}_{\tau-}\right] .
$$

The following result is so-called Clark-Haussmann-Ocone formula.

Proposition 41. Let $\xi \in \mathbb{D}^{1,2}$, and suppose that a process with conditionally independent increments $L_{t}, t \in[0 ; 1]$ has the form

$$
L_{t}=L_{t}^{c}+\int_{0}^{t} \int_{|x| \leq 1} x(N(d s d x)-\nu(d s d x))+\int_{0}^{t} \int_{|x|>1} x N(d s d x) .
$$

Then

$$
\xi=\mathrm{E}[\xi \mid \mathcal{H}]+\int_{0}^{1} p\left(D_{t, 0} \xi\right) d L_{t}^{c}+\int_{0}^{1} \int_{\mathbb{R}_{0}} p\left(D_{t, x} \xi\right)(N(d t d x)-\nu(d t d x)) .
$$


Proof. Let $\xi \in \mathbb{D}^{1,2}$ have an expansion $\xi=\sum_{n=0}^{\infty} I_{n}\left(f_{n}\right)$. Using (41) and (44) we have

$$
\mathrm{E}\left[D_{t, x} \xi \mid \mathcal{F}_{t}\right]=\sum_{n=1}^{\infty} n \mathrm{E}\left[I_{n-1}\left(f_{n}(\cdot, t, x)\right) \mid \mathcal{F}_{t}\right]=\sum_{n=1}^{\infty} n I_{n-1}\left(f_{n}(\cdot, t, x) \mathbf{1}_{[0 ; t]}^{\otimes(n-1)}\right) .
$$

It follows from the arguments in the beginning of the Section 3, that

$$
f_{n}(\cdot, t, x) \mathbf{1}_{[0 ; t]}^{\otimes(n-1)}=f_{n}(\cdot, t, x) \mathbf{1}_{[0 ; t)}^{\otimes(n-1)}
$$

as elements of $L^{2}\left(M_{\pi}^{n-1}\right)$. Thus

$$
\mathrm{E}\left[D_{t, x} \xi \mid \mathcal{F}_{t}\right]=\sum_{n=1}^{\infty} n I_{n-1}\left(f_{n}(\cdot, t, x) \mathbf{1}_{[0 ; t]}^{\otimes(n-1)}\right)=\sum_{n=1}^{\infty} n I_{n-1}\left(f_{n}(\cdot, t, x) \mathbf{1}_{[0 ; t)}^{\otimes(n-1)}\right) .
$$

Since $I_{n-1}\left(f_{n}(\cdot, t, x) \mathbf{1}_{[0 ; t)}^{\otimes(n-1)}\right)$ is predictable then it is easy to show that ${ }^{p}\left(I_{n-1}\left(f_{n}(\cdot, t, x)\right)\right)=I_{n-1}\left(f_{n}(\cdot, t, x) \mathbf{1}_{[0 ; t)}^{\otimes(n-1)}\right)$. Hence

$$
{ }^{p}\left(D_{t, x} \xi\right)=\sum_{n=1}^{\infty} n^{p}\left(I_{n-1}\left(f_{n}(\cdot, t, x)\right)\right)=\sum_{n=1}^{\infty} n I_{n-1}\left(f_{n}(\cdot, t, x) \mathbf{1}_{[0 ; t)}^{\otimes(n-1)}\right) .
$$

Therefore ${ }^{p}\left(D_{t, x} \xi\right)=\mathrm{E}\left[D_{t, x} \xi \mid \mathcal{F}_{t}\right]$ as an $L^{2}\left(M_{\pi}\right)$ processes.

Set $\phi(t, x)=\mathrm{E}\left[D_{t, x} \mid \mathcal{F}_{t}\right]$ and $\psi(t, x)={ }^{p}\left(D_{t, x} \xi\right)$. Then from equality (47) we deduce

$$
\delta(\psi)=\delta(\phi)=\sum_{n=1}^{\infty} I_{n}\left(f_{n}\right)=\xi-\mathrm{E}[\xi \mid \mathcal{H}],
$$

which shows the desired result because Proposition 40 implies that

$$
\delta(\psi)=\int_{0}^{1} p\left(D_{t, 0} \xi\right) d L_{t}^{c}+\int_{0}^{1} \int_{\mathbb{R}_{0}} p\left(D_{t, x} \xi\right)(N(d t d x)-\nu(d t d x)) .
$$

\section{Acknowledgments}

I would like to thank Bernt Øksendal for his encouragement and interest, Paul Kettler for the attentive reading and the Department of Mathematics, University of Oslo, for its warm hospitality. This work was supported by INTAS grant 03-55-1861.

\section{References}

1. Bass, R.F., Cranston, M.: The Malliavin calculus for pure jump Lévy processes and applications to local time. Ann. Probab. 14 (1986), pp. 490-532.

2. Benth,F.E., Di Nunno,G., Løkka, A., Øksendal, B., Proske, F.: Explicit representation of the minimal variance portfolio in markets driven by Lévy processes. Math. Finance 13 (2003), pp. 54-72. 
3. Benth, F.E., Løkka, A.: Anticipative calculus for Lévy processes and stochastic differential equations. Stoch. Stoch. Rep. 76 (2004), no. 3, 191-211.

4. Bichteler, K., Gravereaux, J.B., Jacod, J.: Malliavin Calculus for Processes with Jumps. Gordon and Breach Science Publisher, New York 1987.

5. Di Nunno, G.: On orthogonal polynomials and the Malliavin derivative for Lévy stochastic measures. Preprint series in Pure Mathematics, University of Oslo, 10, 2004.

6. Di Nunno, G.: Random Fields: Skorohod integral and Malliavin derivative. Preprint series in Pure Mathematics, University of Oslo, 36, 2004.

7. Di Nunno, G., Meyer-Brandis, T., Øksendal, B., Proske, F.: Malliavin calculus and anticipative Itô formulae for Lévy processes. Infin. Dimens. Anal. Quantum Probab. Relat. Top. 8 (2005), no. 2, 235-258.

8. Di Nunno, G., Øksendal, B., Proske, F.: White noise analysis for Lévy processes. J. Funct. Anal. 206 (2004), no. 1, 109-148.

9. Engel, D.D.: The multiple stochastic integral. Mem. Amer. Math. Soc. 265 (1982).

10. Itô, K.: Multiple Wiener integral. J. Math. Soc. Japan. 3 (1951), pp. 157-169.

11. Itô, K.: Spectral type of the shift transformation of differential processes with stationary increments. Trans. Am. Math. Soc. 81 (1956), pp. 253-263.

12. Jacod, J., Shiryaev, A.N.: Limit theorems for stochastic processes. 2nd edition. Grundlehren der Mathematischen Wissenschaften [Fundamental Principles of Mathematical Sciences], 288. Springer-Verlag, Berlin, 2003.

13. Kabanov, Yu.M.: On extended stochastic integrals. Th. Probab. Appl. 20 (1975), pp. $710-722$.

14. Karatzas, I., Ocone, D.L.; A generalized Clark representation formula, with application to optimal portfolios. Stochastics Stochastics Rep. 34 (1991), no. 3-4, 187-220.

15. Kulik, A.M.: Malliavin calculus for functionals with generalized derivatives and some applications to stable processes. Ukrainian Math. J. 54 (2002), no. 2, 266279 .

16. Léon, J.A., Solé, J.L., Utzet, F., Vives, J.: On Lévy processes, Malliavin calculus and market models with jumps. Finance Stochast. 6 (2002), pp. 197-225.

17. Liptser, R. Sh., Shiryayev, A. N.: Theory of martingales. Mathematics and its Applications (Soviet Series), 49. Kluwer Academic Publishers Group, Dordrecht, 1989.

18. Løkka, A.: Martingale representation and functionals of Lévy processes. Stochastic Anal. Appl. 22 (2004), no. 4, 867-892.

19. Ma, J., Protter, P., San Martin, J.: Anticipating integrals for a class of martingales. Bernoulli 4 (1998), no.1, pp. 81-114.

20. Malliavin, P.: Stochastic Analysis. Springer-Verlag, New York 1997.

21. Meyer, P.A. Un cours sur les inteégrales stochastiques. Séminare de Probabilités X, Lect. Math. Notes, Vol. 511, Springer Verlag 1976, pp. 245-400.

22. Nualart, D.: The Malliavin Calculus and Related Topics. Springer, Berlin 1995.

23. Nualart, D., Schoutens, W.: Chaotic and predictable representations for Lévy processes. Stochastic Process. Appl. 90 (2000), pp. 109-122.

24. Nualart, D., Vives, J.: Anticipating calculus for the Poisson process based on the Fock space. Séminare de Probabilités XXIV, Lect. Math. Notes, Vol. 1426, Springer Verlag 1990, pp. 154-165.

25. Øksendal, B., Proske, F.: White noise of Poisson random measures. Potential Anal. 21 (2004), no. 4, 375-403. 
26. Picard, J.: On the existence of smooth densities for jump processes. Probab. Theory Rel. Fields 105 (1996), pp. 481-511.

27. Pollard, P: A user's guide to measure theoretic probability.Cambridge Series in Statistical and Probabilistic Mathematics, 8. Cambridge University Press, Cambridge, 2002.

28. Privault, N.: An extension of stochastic calculus to certain non-Markovian processes. Preprint. (1997).

29. Russo, F., Valois, P.: Product of two multiple stochastic integrals with respect to a normal martingale. Stoch. Proc. and Appl. 73 (1998), pp. 47-68.

30. Skorohod, A.V.: On generalization of a stochastic integral. Theory Probab. Appl. 20 (1975), pp. 219-233.

31. Solé, J.L., Utzet, F. and Vives,J.: Canonical Lévy process and Malliavin calculus. Preprint. (2005).

32. Surgailis, D.: On $L^{2}$ and non- $L^{2}$ multiple stochastic integration. Lecture Notes in Control and Information Sci., Vol. 36, Springer, Berlin-New York, 1981, pp. 212-226.

33. Yablonski, A.: The calculus of variations for processes with independent increments. Preprint series in Pure Mathematics, University of Oslo, 15, 2004. 